\title{
MRPLI 3 Promotes Tumor Cell Proliferation, Migration and EMT Process in Breast Cancer Through the PI3K-AKT-mTOR Pathway
}

This article was published in the following Dove Press journal: Cancer Management and Research

\author{
Miaomiao Cai (1D' \\ Hanning $\mathrm{Li}^{2}$ \\ Runfa Chen' \\ Xiang Zhou' \\ 'College of Life Sciences and Health, \\ Wuhan University of Science and \\ Technology, Wuhan, Hubei, 430065, \\ People's Republic of China; ${ }^{2}$ Department \\ of Thyroid and Breast Surgery, Tongji \\ Hospital, Tongji Medical College, \\ Huazhong University of Science and \\ Technology, Wuhan, Hubei, 430030, \\ People's Republic of China
}

Purpose: Breast cancer (BC), with varying histopathology, biology and response to systemic treatment, is the second leading cause of cancer-related mortality. Previous studies have inferred that the expression of mitochondrial ribosomal proteins (MRPs) is possibly related to the occurrence/progression of BC. MRPL13 might be one of the potential MRP candidates that are involved in $\mathrm{BC}$ tumorigenesis, but its role in $\mathrm{BC}$ has rarely been reported. The purpose of the current study was to evaluate the prognostic significance of MRPL13, as well as to explore its potential biological functions in BC.

Materials and Methods: A series of bioinformatic and statistical methods were adopted to assess the MRPL13 expression profile, its relationship with clinicopathological characteristics, copy number variation (CNV), impact on clinical outcomes and relevant functions. All the results are analysed by 1097 BC patients collected from The Cancer Genome Atlas (TCGA) dataset and 52 clinical samples for immunohistochemistry (IHC) assay.

Results: The results demonstrated that the expression of MRPL13 in BC tissues was remarkably elevated than that in normal breast tissues. In addition, the Kaplan-Meier curves and Cox model indicated that patients with high MRPL13 expression were connected to a worse prognosis, heralding the independent prognostic value of this protein in $\mathrm{BC}$. Moreover, an enrichment analysis showed that MRPL13 was mainly involved in cell cycle/division-related, RNA processing (degradation/splicing), MYC targets and the MTORC1 pathways. In addition, RNA interference (RNAi)-mediated MRPL13 silencing remarkedly inhibited proliferation and migration as well as the expression of EMT-related genes of BC cells in vitro. Mechanistically, attenuation of MRPL13 significantly suppressed the phosphorylation of AKT and mTOR, which could be partially abolished by 740Y-P (a PI3K agonist).

Conclusion: Our results provide evidence for the first time that increased MRPL13 expression correlates with adverse clinicopathological variables and unfavorable clinical outcomes of BC patients. Knockdown of MRPL13 restrains the proliferation and migration potential and EMT process of BC through inhibiting PI3K/AKT/mTOR signaling pathway.

Keywords: breast cancer, MRPL13, prognosis, biomarker, PI3K-AKT-mTOR

\section{Introduction}

Breast cancer (BC) has remained the most frequently diagnosed malignancy and one of the leading causes of cancer-related deaths among females worldwide. ${ }^{1}$ The most widely used clinical classification of $\mathrm{BC}$ is based on the expression of progesterone receptor (PR), estrogen receptor (ER), and human epidermal growth factor receptor 2 (Her-2), which are considered as classical prognostic indicators
College of Life Sciences and Health,

Wuhan University of Science and

Technology, Wuhan, Hubei, 430065 ,

People's Republic of China

Tel + I3699206313

Email zhouxiang@wust.edu.cn

Cancer Management and Research 2021:13 2009-2024 
and therapeutic targets. ${ }^{2,3}$ Despite limited progress in $\mathrm{BC}$ treatment with drugs against these targets, such as anti-Her -2 targeted therapy (trastuzumab), some patients are still predisposed to advanced stages attributing to the strong heterogeneity of tumors. ${ }^{4,5}$ In 2012, the mammalian target of mTOR inhibitor everolimus has been approved as a treatment of advanced BC in patients. ${ }^{6}$ Much work has focused on the PI3K/AKT/mTOR pathway because of its key role in various oncogenic processes. ${ }^{7}$ Aberrant mTOR pathway was regarded as an activation of driving the different molecular subtypes in $\mathrm{BC}$ like ligandindependent ER activation without traditional binding or regulation of estrogen. ${ }^{8}$ Therefore, it is crucial to further investigate the pathogenesis as well as identify more reliable molecular markers of targeting mTOR/AKT in BC.

Mitochondrial ribosomal protein L13 (MRPL13), encoding the mitochondrial ribosomal 39S large subunit protein, locates on human chromosome 6. MRPL13 belongs to the primary binding proteins, being more advanced in the ribosome self-assembly process and rRNA binding sequence. ${ }^{9}$ Mammalian mitochondrial ribosomes containing small $28 \mathrm{~S}$ and large $39 \mathrm{~S}$ subunits are composed of 30 and 52 different mitochondrial ribosomal proteins (MRPs) and encoded by the nuclear genome. ${ }^{10,11}$ Over the past decade, the MRPs-induced cancer cell apoptosis and proliferation via mitochondrial translation and mitotic ribosomal proteins has been well documented. ${ }^{12,13}$ For instance, MRPL41 maintains p53 stability and promotes p53-induced apoptosis in response to growth inhibition conditions. It also induces a G1 phase cell cycle arrest by stabilizing the p27 (Kip1) protein in p53-deleted cells. ${ }^{14}$ Mitochondrial dysfunction caused by MRPL33-L silencing, leading to increased reactive oxygen accumulation and reduced ATP production, is intensely linked to colorectal cancer tumorigenesis. ${ }^{15}$ Meanwhile, evidence from GeneChip comparing the gene expression profiles between androgen-dependent and androgenindependent prostate cancer demonstrated that MRPL20 exhibited strikingly different expression levels in these two phenotypes, heralding a potential role of it in androgen resistance. ${ }^{16}$ However, the functional effects of MRPL13 in BC and its potential association with prognosis remain obscure.

In this study, we explored the expression pattern, prognostic value as well as the association of clinicopathological characteristics of MRPL13 in BC through comprehensive bioinformatics research, and elucidated its potential mechanism of action in BC. We substantiated that the differential expression patterns of MRPL13 are tightly associated with tumor progression and clinical outcomes. Ectopic expression of MRPL13 triggers wide-ranging cellular processes including DNA repair, G2M checkpoint control, cell cycle regulation, and RNA degradation, and so on. More importantly, the data derived from in vitro assays indicated that knockdown of MRPL13 hampers the proliferation and migration ability and impedes EMT process of $\mathrm{BC}$ cell lines through the $\mathrm{PI} 3 \mathrm{~K} / \mathrm{AKT} / \mathrm{mTOR}$ pathway. Our results shed new light on the understanding of the MRPL13-related mechanism in the development of BC.

\section{Materials and Methods Clinical Samples}

Human $\mathrm{BC}$ and adjacent normal breast tissues for immunohistochemistry analysis were collected from 52 patients treated in Tongji Hospital (Wuhan, China). Patients in this study had not received any treatment like chemotherapy, radiotherapy or targeted therapy before surgery. Informed consent documents were signed from all participants and the experimental protocols were approved by the Ethics Committee of Wuhan University of Science and Technology. Moreover, the research design and procedure strickly adhere to the principles outlined in the Declaration of Helsinki.

\section{Cell Culture and Transfection}

Human breast cancer cell lines MDA-MB-231 and T47D were obtained from the American Type Culture Collection (ATCC; Manassas, VA, USA). MDA-MB-231 cells were maintained in DMEM (Gibco, USA) and T47D cells in RPMI-1640 medium (Gibco, USA), supplemented with $10 \%$ fetal bovine serum (FBS; Gibco, USA) and 1\% antibiotic/antimycotic solution and incubated in an incubator with $5 \% \mathrm{CO} 2$ and $100 \%$ humidity at $37^{\circ} \mathrm{C}$.

MRPL13 siRNAs $(100 \mu \mathrm{M})$ were transfected into MDA-MB-231 and T47D cells with lipo3000 (Invitrogen) according to the manufacturer instructions. For pathway analysis, cells were pre-incubated with 20 $\mu \mathrm{g} / \mathrm{mL}$ of 740Y-P (Selleck Chemicals, S7865) for $2 \mathrm{hr}$. Knockdown efficiencies were confirmed by Western blot and real-time PCR after cell transfection for $48 \mathrm{hr}$ or $72 \mathrm{hr}$.

\section{Data Sources and Preparation}

The original datasets of $1097 \mathrm{BC}$ patient demographic features and clinicopathological parameters were downloaded from The Cancer Genome Atlas (TCGA) dataset (https://portal.gdc.cancer.gov/). Information of these 
patients including age at initial diagnosis, histological type, Tumor-Node-Metastasis (TNM) stage and molecular markers (ER, PR and Her-2), etc. was detailed in Table 1. The mRNA expression values of MRPL13 (Reads Per Kilobase Million, FPKM) were extracted from raw data files, followed by normalization and $\log 2$ conversion for further analyses.

Additionally, four microarray series, including GSE71053, ${ }^{17}$ GSE120129, ${ }^{18}$ GSE50567 ${ }^{19}$ and GSE21422, ${ }^{20,21}$ were retrieved from the Gene Expression Omnibus (GEO) database (http://www.ncbi.nlm.nih.gov/ geo). The details of these series are summarized in Table 2 .

\section{Survival Analysis by Kaplan-Meier Curves and Cox Regression Analyses}

Kaplan-Meier (K-M) curves of overall survival (OS), recurrence-free survival (RFS), and distant metastasisfree survival (DMFS) were generated using the K-M plotter (http://kmplot.com/analysis/index.php?p=ser vice), a comprehensive database for evaluating the effect of specified genes on the survival of a lot of cancers including breast cancer. ${ }^{22}$ Additionally, K-M survival analysis of OS and RFS in subgroups of different clinicopathological characteristics (eg, patient's age, clinical stage and molecular subtypes) was carried out using TCGA-BRCA cohort. Specifically, individuals were classified into high and low expression groups in accordance with the median MRPL13 expression level. The hazard ratio (HR) with $95 \%$ confidence interval (CI) was then calculated and a P-value indicating statistically significant differences was generated by Log rank test.

In addition, univariate followed by multivariate Cox proportional hazard regression models were carried out to identify clinicopathological characteristics for OS based on the TCGA cohort. The results were displayed as HR with $95 \%$ CI. Values of $\mathrm{P}<0.05$ were considered as statistically significant for the above analyses.

\section{Copy Number Variation Analysis}

The copy number variation (CNV) of MRPL13 gene was determined by querying the cBio Cancer Genomics Portal (cBioPortal) (http://www.cbioportal.org) with METABRIC data set. ${ }^{23}$ Types of CNV include shallow deletion, diploid, gain and amplification. The relationships between the CNV and the clinicopathological characteristics (including tumor grade, stage, cell proliferation ability) were expressed as discrete graphs and stacked histograms. Moreover, K-M method
Table I The Breast Cancer Patient's Demographic Features and Clinicopathological Parameters from TCGA Database

\begin{tabular}{|c|c|c|}
\hline Characteristics & Total No. & No. of Patients \\
\hline Age (year) & 1097 & \\
\hline$>50$ & & 331 \\
\hline$\leq 50$ & & 766 \\
\hline Histological type & 1056 & \\
\hline Infiltrating ductal carcinoma & & 784 \\
\hline Infiltrating lobular carcinoma & & 203 \\
\hline Medullary carcinoma & & 6 \\
\hline Mucinous carcinoma & & 17 \\
\hline Other, specify & & 46 \\
\hline Clinical stage & 1073 & \\
\hline I & & 183 \\
\hline II & & 621 \\
\hline III & & 249 \\
\hline IV & & 20 \\
\hline T stage & 1094 & \\
\hline TI & & 281 \\
\hline $\mathrm{T} 2$ & & 635 \\
\hline T3 & & 138 \\
\hline T4 & & 40 \\
\hline $\mathrm{N}$ stage & 1077 & \\
\hline No & & 516 \\
\hline $\mathrm{NI}$ & & 364 \\
\hline N2 & & 120 \\
\hline N3 & & 77 \\
\hline Distant metastasis & 934 & \\
\hline Mo & & 912 \\
\hline MI & & 22 \\
\hline ER status & 984 & \\
\hline Negative & & 228 \\
\hline Positive & & 756 \\
\hline PR status & 981 & \\
\hline Negative & & 325 \\
\hline Positive & & 656 \\
\hline Her-2 status & 687 & \\
\hline Negative & & 532 \\
\hline Positive & & 155 \\
\hline Classical molecular subtype & 719 & \\
\hline Luminal & & 566 \\
\hline Her-2 overexpression & & 37 \\
\hline TNBC & & 116 \\
\hline
\end{tabular}

Abbreviations: ER, estrogen receptor; PR, progesterone receptor; Her-2, human epidermal growth factor receptor-2; TNBC, triple-negative breast cancer.

was used to explore the impact of MRPL13 CNV on OS in BC patients and the result was depicted as a K-M plot curve with a log-rank P-value. 
Table 2 Characteristics of GEO Series Included in This Study

\begin{tabular}{|l|l|l|l|l|l|}
\hline GEO Series & Contributor(s) & N & T & Country & Platform \\
\hline GSE71053 & Thomassen M, 20I8 & 12 & 6 & Denmark & Affymetrix Human Genome UI33 Plus 2.0 Array \\
GSEI20129 & Ernlund A, 20I8 & 78 & 30 & USA & Affymetrix Human Genome UI33 Plus 2.0 Array \\
GSE42568 & Schaefer C, 20II & 5 & 14 & Ireland & Affymetrix Human Genome UI33 Plus 2.0 Array \\
GSE21422 & Lisowska K, 20II & 6 & 35 & Germany & Affymetrix Human Genome UI33 Plus 2.0 Array \\
\hline
\end{tabular}

Abbreviations: GEO, Gene Expression Omnibus; T, tumor; N, normal.

\section{Gene Set Enrichment Analysis}

To further explore the mechanism of MRPL13 in the pathogenesis of BC, gene set enrichment analysis (GSEA) (http:// www.broadinstitute.org/gsea/index.jsp), ${ }^{24}$ a computational means to determine whether a prior-defined gene set shows statistical significance or consistent differences across two biological patterns, was carried out with the TCGA breast cancer cohort. In this study, BC samples were first classified into high to low levels according to their MRPL13 expression values, with the top third as the high-MRPL13 expression group and the bottom third as the low-MRPL13 expression group. Next, GSEA was conducted using GSEA v4.0.1. The gene sets, including Kyoto Encyclopedia of Genes and Genomes (KEGG) (c2.cp.kegg.v7.0.symbols.gmt) and Hallmarks (h.all.v7.0.symbols.gmt) pathways, were used to determine and explore the predefined biological states or processes. To obtain significant enrichment, the threshold for statistical significance was set to the absolute normalized enrichment score (NES) values exceeding 2 combined with nominal $\mathrm{P}$-value $<0.01$ and false discovery rate $(\mathrm{FDR})<0.05$.

\section{RNA Isolation and Quantitative Real-Time PCR (qRT-PCR)}

TRIzol reagent (Invitrogen, USA) was used to extract the total RNA from the cultured cells. For mRNA expression analysis, cDNA was synthesized by reverse transcription with $1 \mu \mathrm{g}$ of total RNA using Evo M-MLK RT Kit with gDNA Clean for qPCR II (AG, China). The qRT-PCR was performed using iTagTM Universal SYBR Green Supermix (BIO-RAD, USA) with a CFX96 ${ }^{\mathrm{TM}}$ Real-Time PCR System (BIO-RAD, CA, USA). Primer Sequences used were listed below: hMRPL13 forward: 5'- ACATAAACCTGTGTACCATGCAC -3', hMRPL13 reverse: 5'-GGTAGCCAGTATGCGAAGAGT -3'; hGAPDH forward: 5'- GGAGCGAGATCCCTCCAA AAT -3', and hGAPDH reverse: 5'- GGCTGTTGT CATACTTCTCATGG - $3^{\prime}$. GAPDH was employed as the internal control. The relative expression level of the detected genes was calculated by $2^{(-\Delta \Delta \mathrm{Ct})}$ method. ${ }^{25}$ Each test was repeated three times.

\section{Western Blotting Assay}

Cells were lysed in RIPA buffer (Beyotime, China) containing PMSF (dilution, 1:50; Beyotime, China). Before denaturation, total protein concentration was determined using BCA protein assay kit (Beyotime, China), according to the manufacturer's instructions. After electrophoretic separation by $10 \%$ SDS-PAGE gel, equal amounts of protein were transferred to polyvinylidene difluoride (PVDF) membranes (Millipore). The information of primary antibodies is shown in Table 3. Finally, the resulting signal was detected using BeyoECL Moon Western chemiluminescent ECL kit (Beyotime, China).

\section{Immunohistochemistry (IHC)}

Tumor and normal tissues were fixed in $4 \%$ paraformaldehyde for $24 \mathrm{hr}$, paraffin embedded, and sectioned at $5 \mathrm{~mm}$. IHC staining of specimens was carried out using the primary antibodies anti-MRPL13 (Proteintech, China); the goat anti-rabbit IgG secondary antibodies (Servicebio, G1216-200T) were used at appropriate concentration. The 3,3'-diaminobenzidine (DAB) solution (Servicebio, G1216-200T) was used for color development. Finally, the nuclei were counterstained with hematoxylin. The evaluation of MRPL13 expression was

Table 3 The Information for Primary Antibodies Used in Western Blotting Assay

\begin{tabular}{|l|c|c|c|}
\hline $\begin{array}{l}\text { Primary } \\
\text { Antibody }\end{array}$ & $\begin{array}{c}\text { Dilution } \\
\text { Concentration }\end{array}$ & Supplier & Code \\
\hline$\beta$-actin & $\mathrm{I}: 6000$ & abcam & ab227387 \\
MRPLI3 & $\mathrm{I}: 1000$ & abcam & abl90232 \\
E-cadherin & $\mathrm{I}: 1000$ & CST & 14,472 \\
Vimentin & $\mathrm{I}: 1000$ & CST & $574 \mathrm{I}$ \\
Snaill & $\mathrm{I}: 1000$ & Proteintech & I3,099-I-AP \\
Snail2 & $\mathrm{I}: 1000$ & Proteintech & I2,I29-I-AP \\
AKT & $\mathrm{I}: 1000$ & CST & 4685 \\
P-AKT & $\mathrm{I}: 1000$ & CST & 4060 \\
mTOR & $\mathrm{I}: 1000$ & CST & 2983 \\
P-mTOR & $\mathrm{I}: 1000$ & CST & 5536 \\
\hline
\end{tabular}

Abbreviations: MRPLI3, mitochondrial ribosomal protein LI3; AKT, serine/threonine-protein kinase; mTOR, mammalian target of rapamycin. 
scored according to the intensity of the dye color ( 0 , negative; 1, weak; 2 , moderate; 3 , strong) and the number of positive cells ( 0 , no staining; $1,<25 \% ; 2,26-50 \% ; 3,51-75 \%$; 4 , $>75 \%$ ). Additionally, patients with high expression of MRPL13 was described as $\mathrm{IHC} \geq 4$, while the low expression was described as $\mathrm{IHC}<4$.

\section{Wound-Healing Assay}

After cell transfection for $48 \mathrm{hr}$, a wound was created by manually scraping the cell monolayer with a $200 \mu \mathrm{L}$ tip in 6 -well plate and the cells were maintained in serum-free medium. Photomicrographs were taken of live cells at $\times 10$ magnification at $12 \mathrm{hr}$ and $36 \mathrm{hr}$.

\section{Cell Proliferation Assay}

The cell proliferation ability was measured with cell counting kit-8 (CCK-8; Vazyme, China). Briefly, the breast cancer cells after a $48 \mathrm{hr}$ transfection of siRNA were seeded in 96-well plates at the density of 2000 cells per well. The optical density value (OD value) was measured at the wavelength of $450 \mathrm{~nm}$ after $3 \mathrm{hr}$ of incubation every $24 \mathrm{hr}$ for 4 days.

\section{Statistical Analysis}

Statistical analyses were completed with $\mathrm{R}$ software ( $\mathrm{R}$ Core Team, Version 3.6.1) or GraphPad Prism (Version 8, San Diego, CA, USA). The global differences of the MRPL13 expression between 1109 BC tissues (from 1097 patients possibly both with primary foci and metastasis of BC) and 113 normal breast tissues were assessed by the Wilcoxon test, while the differential expression of 113 paired tumor and normal samples was determined by the paired two-tailed $t$-test. Box plots were depicted utilizing "ggplot2" and "beeswarm" $\mathrm{R}$ packages. Logistic regression was carried out to explore the association of clinical features with the MRPL13 expression, in where the odds ratios (OR) with 95\% CI were decided for each specific clinical variable. The analyses for in-vitro assay were performed by using an un-paired Student's $t$-test. $\mathrm{P}<0.05$ was set as
A

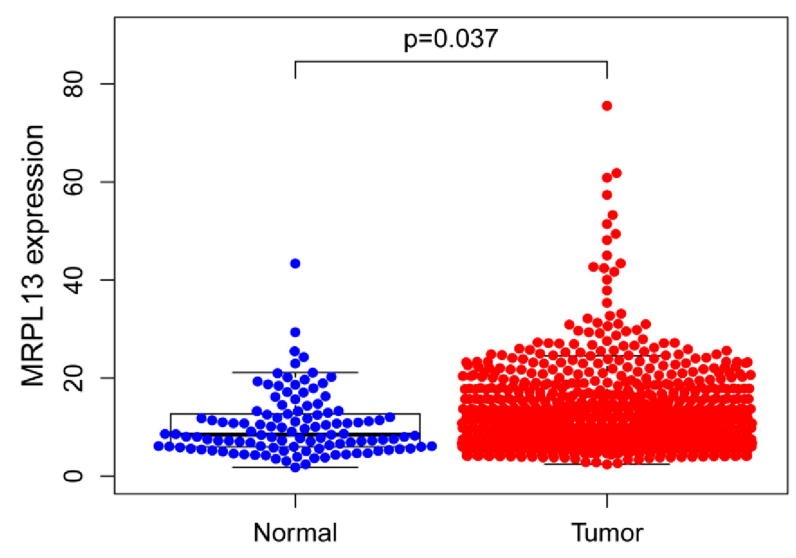

C

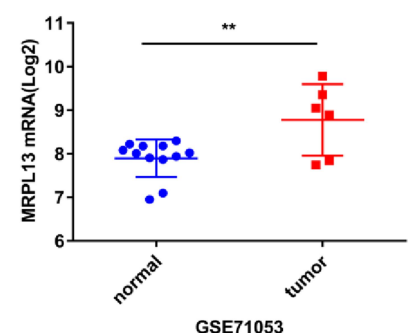

GSE71053

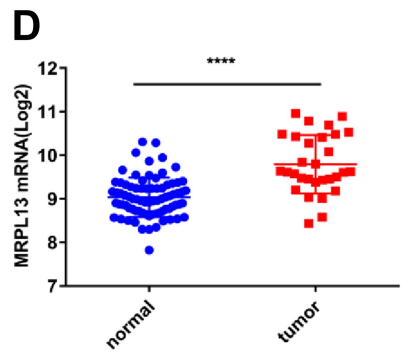

GSE120129
B

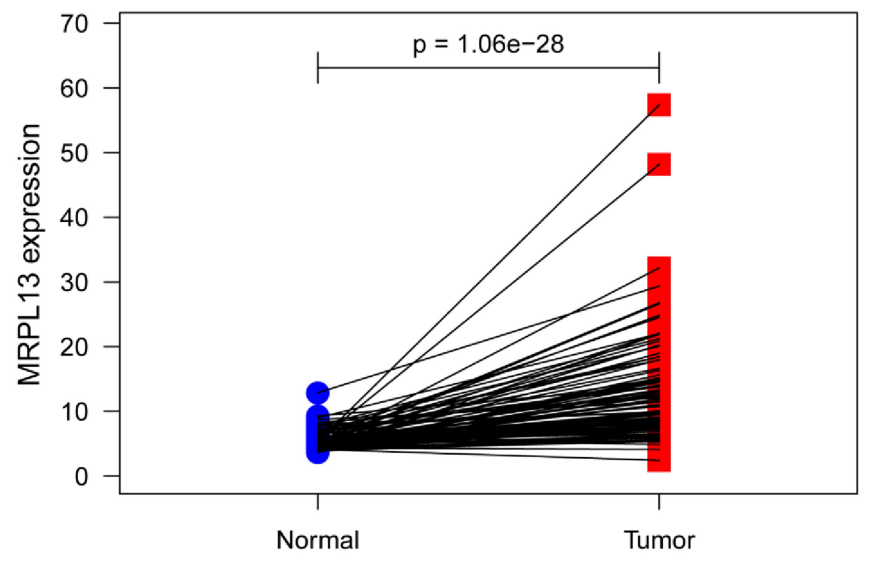

E

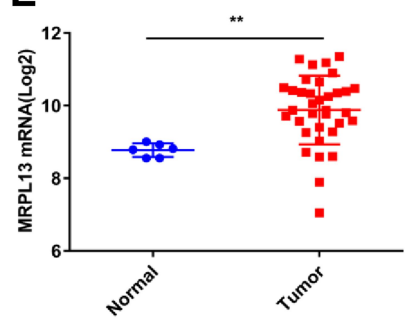

GSE50567

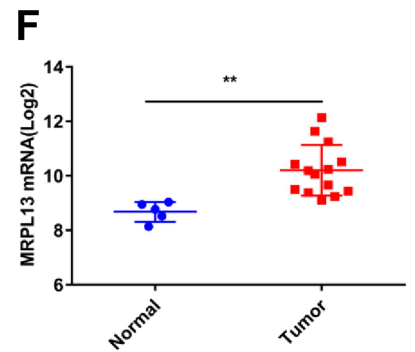

GSE21422

Figure I MRPLI 3 mRNA expression in BC. (A) Comparison of MRPLI 3 mRNA expression in BC and non-cancerous tissues in TGCA dataset. (B) Comparison of MRPLI 3 mRNA expression in 113 paired $B C$ and adjacent non-cancerous tissues in TCGA dataset. Comparison of MRPLI 3 mRNA expression in BC and non-cancerous tissues in GEO series: GSE7I053 (C), GSEI 20129 (D), GSE50567 (E), GSE2I422 (F). **p<0.0I; ****p<0.000I.

Abbreviations: MRPLI3, mitochondrial ribosomal protein LI3; BC, breast cancer; TCGA, The Cancer Genome Atlas; GEO, Gene Expression Omnibus; GSE, GEO series. 
A

\section{Tumor}

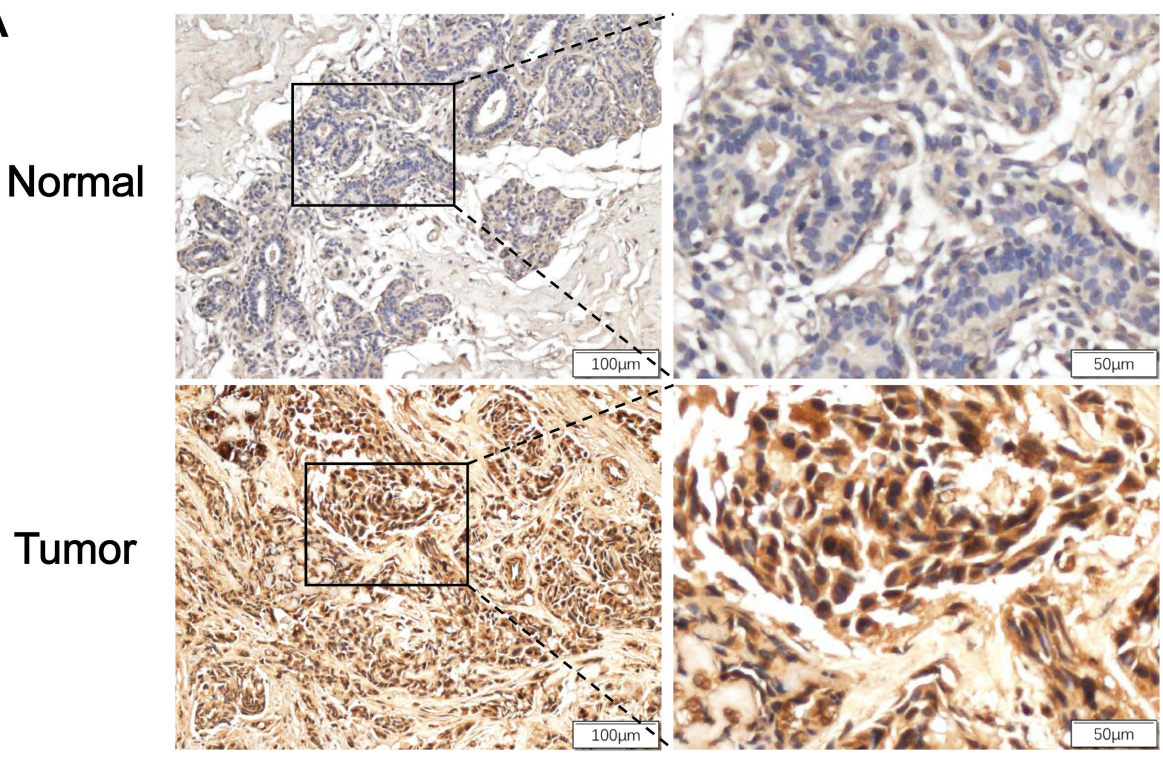

B

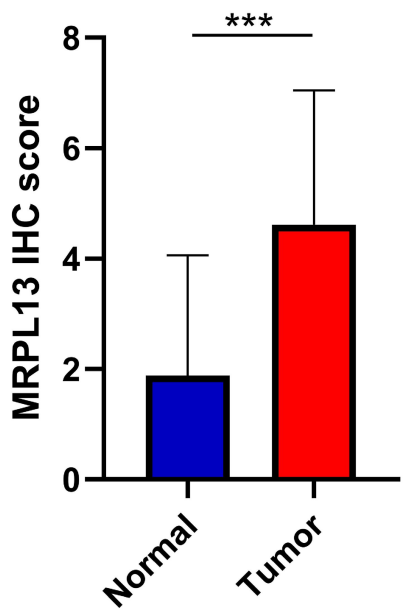

Figure 2 Assessment of the protein expression of MRPLI 3 in BC tissues and adjacent controls by IHC. (A) Representative photographs of MRPLI 3 staining in BC and adjacent normal breast tissues. (B) Histogram shows IHC score quantification of MRPLI 3 in BC and normal tissue groups ( $\mathrm{n}=52$ ). $* * * p<0.001$.

Abbreviation: IHC, immunohistochemistry.

the threshold indicating statistical significance for all above analyses.

Table 4 Correlation of MRPLI3 Expression* with Clinicopathological Characteristics of Breast Cancer, Based on the Logistic Regression Analysis

\begin{tabular}{|c|c|c|c|}
\hline $\begin{array}{l}\text { Clinical } \\
\text { Characteristics }\end{array}$ & $\begin{array}{l}\text { Total } \\
(\mathbf{N})\end{array}$ & $\begin{array}{l}\text { Odds Ratio in } \\
\text { MRPLI } 3 \text { Expression }\end{array}$ & P-value \\
\hline Age $(\leq 50$ vs $>50)$ & 1097 & $1.13(0.97-1.99)$ & 0.231 \\
\hline Stage $(I+I I$ vs III+IV) & 1073 & $0.72(0.46-1.22)$ & 0.003 \\
\hline $\mathrm{T}(\mathrm{TI}+2 \mathrm{vs} \mathrm{T} 3+4)$ & 1094 & $0.82(0.5 I-I .49)$ & 0.026 \\
\hline $\mathrm{N}(\mathrm{N} 0$ vs $\mathrm{NI}+2+3)$ & 1077 & $0.95(0.67-1.55)$ & 0.412 \\
\hline$M(M 0$ vs $M I)$ & 934 & $0.36(0.28-0.87)$ & $<0.001$ \\
\hline $\begin{array}{l}\text { Histological type } \\
\text { (IDC vs ILC) }\end{array}$ & 987 & $1.65(1.09-2.87)$ & 0.035 \\
\hline $\begin{array}{l}\text { ER status (negative } \\
\text { vs positive) }\end{array}$ & 984 & $1.39(0.97-2.44)$ & 0.006 \\
\hline $\begin{array}{l}\text { PR status (negative } \\
\text { vs positive) }\end{array}$ & 981 & $1.41(1.01-2.89)$ & 0.027 \\
\hline $\begin{array}{l}\text { Her-2 status } \\
\text { (negative vs positive) }\end{array}$ & 687 & 0.91 (0.67-1.83) & 0.107 \\
\hline $\begin{array}{l}\text { TNBC (non-TNBC } \\
\text { vs TNBC) }\end{array}$ & 719 & $0.7 \mathrm{I}(0.44-1.30)$ & 0.002 \\
\hline
\end{tabular}

Note: *Categorical dependent variable, greater or less than the median MRPLI3 expression level.

Abbreviations: IDC, infiltrating ductal carcinoma; ILC, infiltrating lobular carcinoma.

\section{Results}

\section{Increased MRPLI 3 Expression in BC Tissues}

The differential expression of MRPL13in BC tissues and adjacent non-cancerous tissues was verified using TCGA and GEO databases. Data from 1109 patient samples of TCGA database indicated that the mRNA expression of MRPL13 in BC tissues was significantly elevated than that in normal breast tissues $(p=0.037$, Figure 1A). Moreover, paired sample analysis also revealed that the mRNA level of MRPL13 was dramatically upregulated in tumor tissues than in noncancerous ones ( $p=1.06 \mathrm{e}-28$, Figure 1B). Consistent with the results in TCGA database, the expression level of MRPL13 was considerably heightened in BC tissues compared to normal tissues in 4 GEO series (GSE71053, GSE120129, GSE50567 and GSE21422, all $p<0.01$, Figure $1 \mathrm{C}-\mathrm{F})$. In addition, IHC assay confirmed that $\mathrm{BC}$ tissues showed higher MRPL13 expression than the matched tumoradjacent control (Figure 2A and $\mathrm{B}, \mathrm{n}=52$ ). These results collectively suggest that the transcription of MRPL13 is elevated in accordance with the clinical status of BC.

\section{Association Between MRPLI3 Expression and Clinicopathological Features of BC Patients}

As shown in Table 4, logistic regression analyses indicated that the patients with higher MRPL13 expression were 

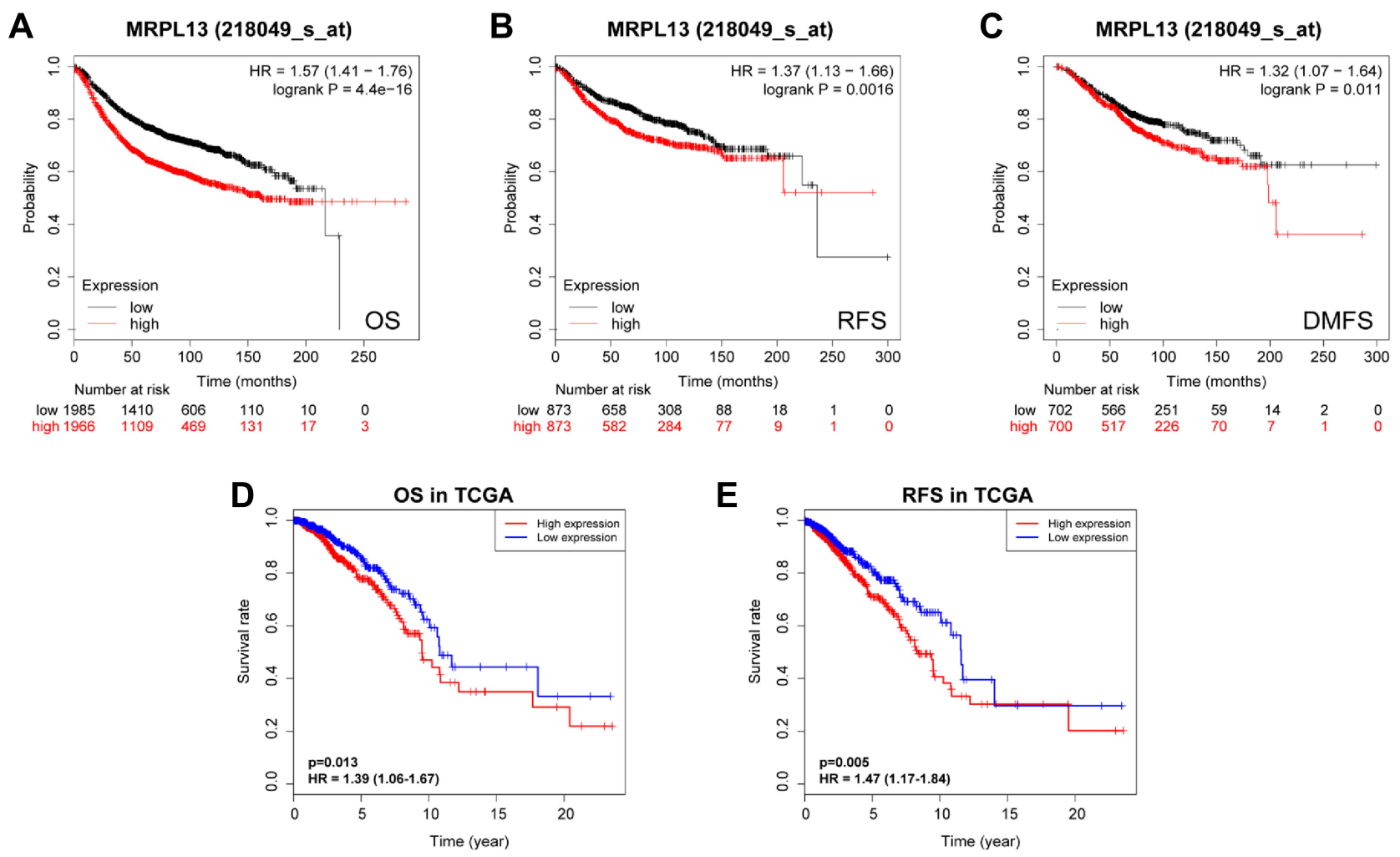

Figure 3 Kaplan-Meier survival analysis of MRPLI 3 mRNA expression in BC patients. Correlation between MRPLI3 and OS (A), RFS (B), and DMFS (C) from Kaplan-Meier plotter database. The impact of MRPLI 3 mRNA expression on OS (D) and RFS (E) of BC patients according to data from TCGA.

Abbreviations: OS, overall survival; RFS, recurrence-free survival; DMFS, distant metastasis-free survival.

present as more advanced clinical stage $(p=0.003)$, $\mathrm{T}$ classification $(p=0.026)$, and distant metastasis $(p<0.001)$. The patients with ER negative $(p=0.006)$, PR negative $(p=0.027)$ and triple-negative breast cancer (TNBC) status $(p=0.002)$ have significantly higher expression of MRPL13; as for histological types, the MRPL13 expression was dramatically higher in invasive ductal carcinoma (IDC) than in invasive lobular carcinoma (ILC) $(p=0.035)$. These results illustrate that patients with a higher expression of MRPL13 suffer from high risks of developing more advanced breast tumors than those with lower MRPL13 expression, inferring a close association of this protein with the clinicopathological features of $\mathrm{BC}$.

\section{MRPLI 3 as an Independent Marker of Unfavorable Prognosis in BC Patients}

$\mathrm{K}-\mathrm{M}$ survival curves from K-M plotter database showed that BC patients with higher MRPL13 abundance correlated with poorer $\mathrm{OS}(\mathrm{HR}=1.57,95 \% \mathrm{CI}=1.41-1.76$, $p=4.4 \mathrm{e}-16), \quad \mathrm{RFS} \quad(\mathrm{HR}=1.37, \quad 95 \% \quad \mathrm{CI}=1.13-1.66$, $p=0.0016)$ and DMFS $(\mathrm{HR}=1.32,95 \% \mathrm{CI}=1.07-1.64$, $p=0.011$ ) (Figure $3 \mathrm{~A}-\mathrm{C}$ ). This result was further confirmed by TCGA cohort, where a shorter $\mathrm{OS}(\mathrm{HR}=1.39, p=0.013)$ and $\mathrm{RFS}(\mathrm{HR}=1.47, p=0.005)$ (Figure $3 \mathrm{D}$ and $\mathrm{E}$ ) time was observed in patients with high level of MRPL13 expression. Furthermore, the subgroup analysis regarding the patient's age, clinical stage and molecular subtypes demonstrated that high MRPL13 expression was significantly correlated with a worse OS in group age $\geq 50$ $(\mathrm{HR}=1.33, \quad p=0.024), \quad \mathrm{IDC} \quad(\mathrm{HR}=1.78, \quad p=0.03), \quad \mathrm{ILC}$ $(\mathrm{HR}=1.49, p=0.003)$, stage $\mathrm{I}+\mathrm{II} \quad(\mathrm{HR}=1.31, p=0.008)$, stage $\mathrm{III}+\mathrm{IV} \quad(\mathrm{HR}=1.85, p=0.002)$, Luminal $(\mathrm{HR}=1.42$, $p=0.004)$ and TNBC $(\mathrm{HR}=1.87, p=0.022)$ subtypes (Figure 4), but the same expression was associated with a poorer RFS in group age $\geq 50(\mathrm{HR}=1.41, p=0.001)$, IDC $(\mathrm{HR}=1.67, p=0.009)$, stage $\mathrm{I}+\mathrm{II} \quad(\mathrm{HR}=1.71, p=7.324 \mathrm{e}-4)$ and Luminal $(\mathrm{HR}=1.34, p=0.013)$ subtype (Figure 5).

Furthermore, the univariate Cox model revealed that the age $\quad(\mathrm{HR}=1.16, \quad p=0.011), \quad$ stage $\quad(\mathrm{HR}=2.19$, $p<0.001), \quad \mathrm{T} \quad(\mathrm{HR}=1.43, \quad p<0.001), \quad \mathrm{N} \quad(\mathrm{HR}=1.63$, $p<0.001)$ and $\mathrm{M}(\mathrm{HR}=4.81, p<0.001)$ classification, and MRPL13 expression $(\mathrm{HR}=1.98, p=0.002)$ represented potential survival-related factors (Table 5, left). Moreover, multivariate cox regression analysis of 


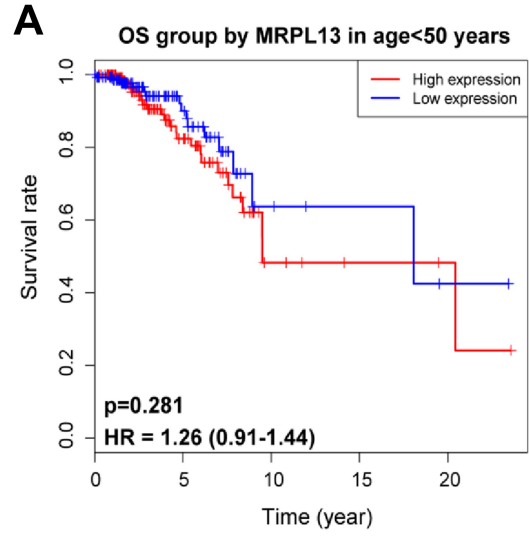

D
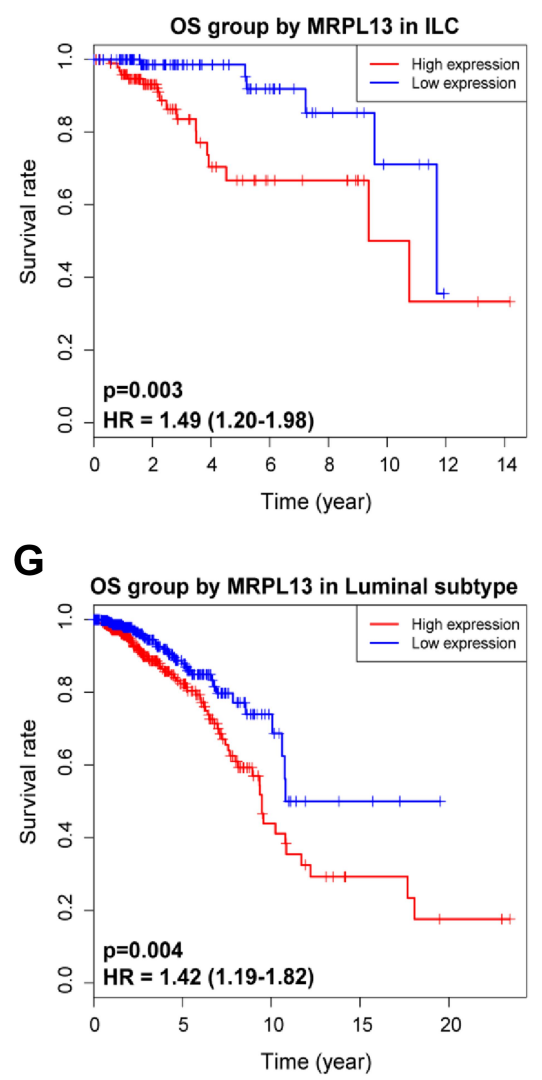

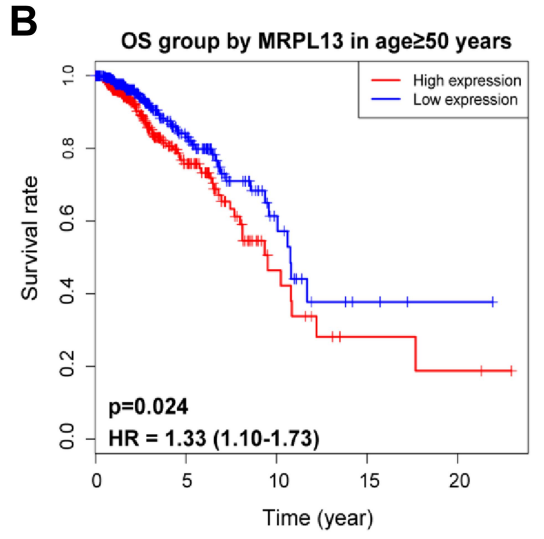

$\mathbf{E}$
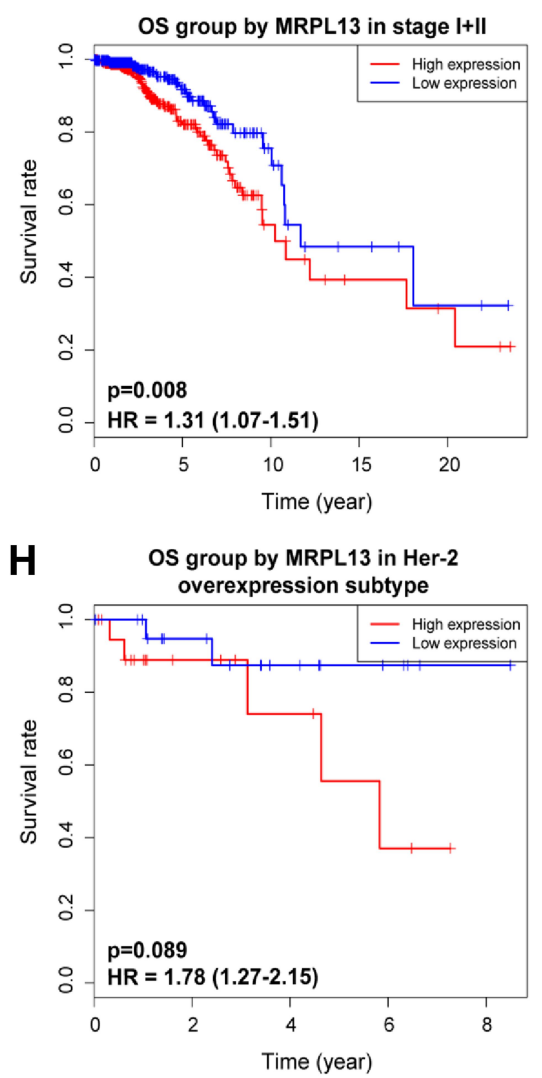

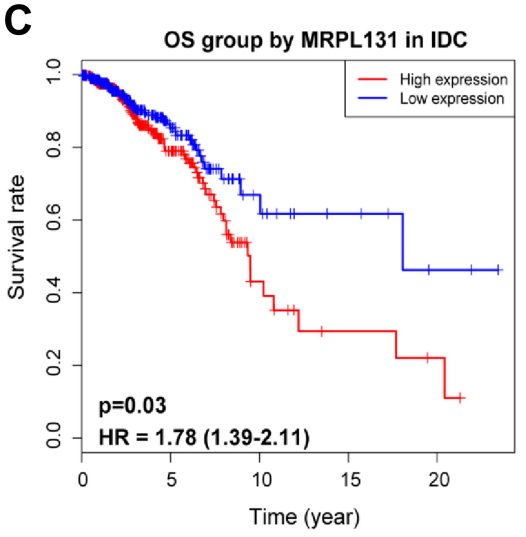

$\mathbf{F}$

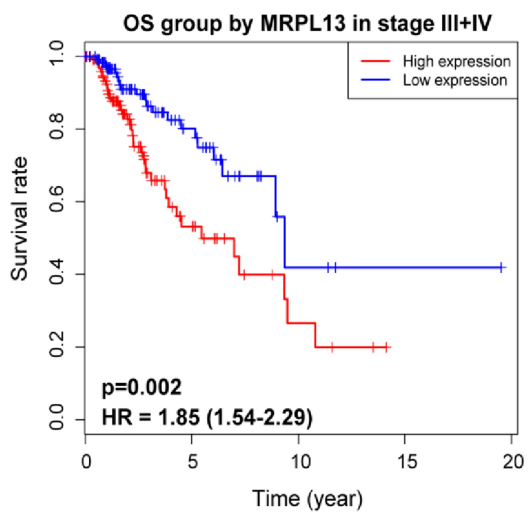

I

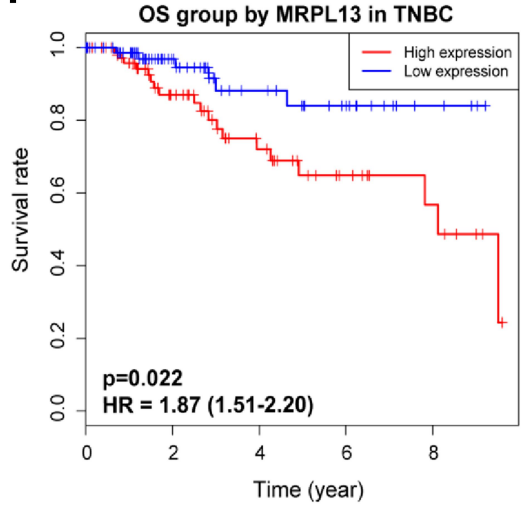

Figure 4 Effect of MRPLI 3 mRNA expression on OS of BC patients stratified by different clinical features. Subgroup analysis of group age $<50$ years $(\mathbf{A})$, $\geq 50$ years (B), IDC (C), ILC (D), stage I+II (E), stage III+IV (F), Luminal (G), Her-2 overexpression (H) and TNBC (I) subtypes.

Abbreviations: IDC, infiltrating ductal carcinoma; ILC, infiltrating lobular carcinoma; Her-2, human epidermal growth factor receptor-2; TNBC, triple-negative breast cancer.

variables significant in the univariate analysis was conducted. The results revealed that the stage $(\mathrm{HR}=1.90$, $p=0.032)$, lymph node involvement $(\mathrm{HR}=1.51$, $p=0.002)$, distant metastasis $(\mathrm{HR}=3.98, p<0.001)$, and increased expression of MRPL13 (HR=1.82, $p=0.006)$ were independent risk determinants that affect the OS of $\mathrm{BC}$ patients (Table 5, right).

\section{Genomic Alterations of MRPLI3 in BC}

$\mathrm{CNV}$ predominate the factors affecting the gene expression level and is linked to a wide range of human conditions, such as cancer, genetic diseases and cardiovascular disorders. ${ }^{26}$ We then used the cBioPortal tool to assess the types and frequency of MRPL3 gene CNV in $\mathrm{BC}$ based on DNA sequencing data from the 


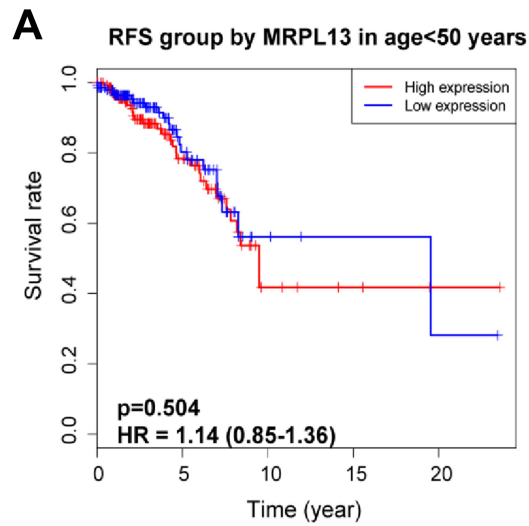

D

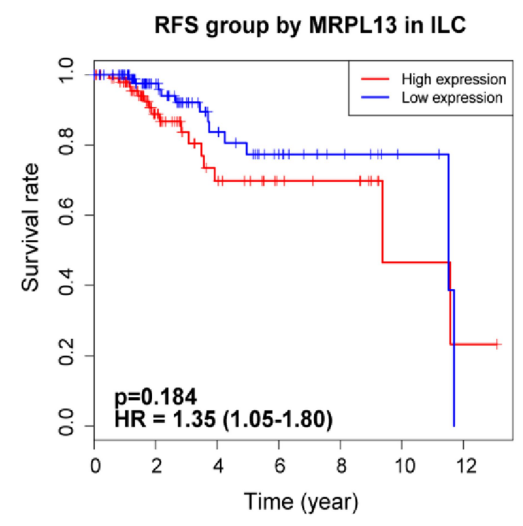

G

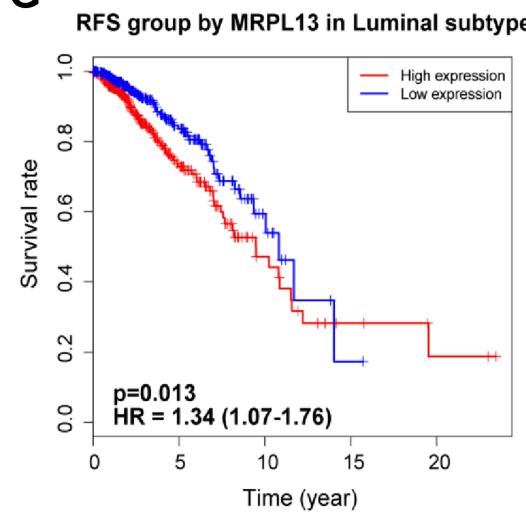

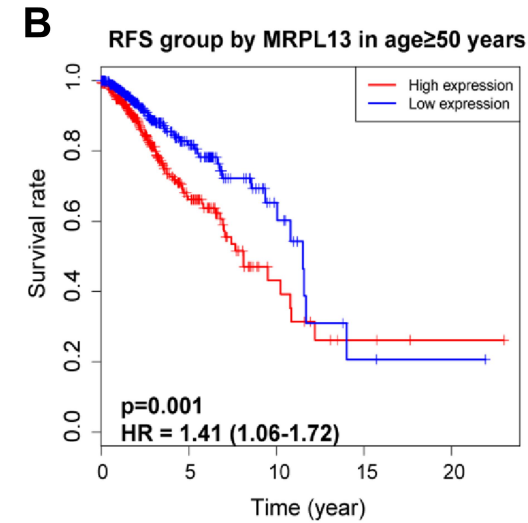

E

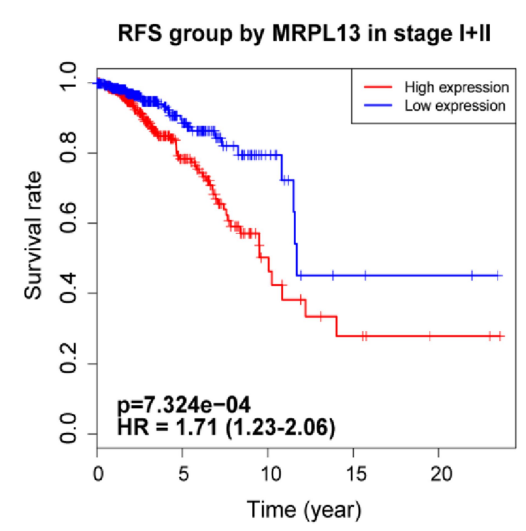

H RFS group by MRPL13 in Her-2

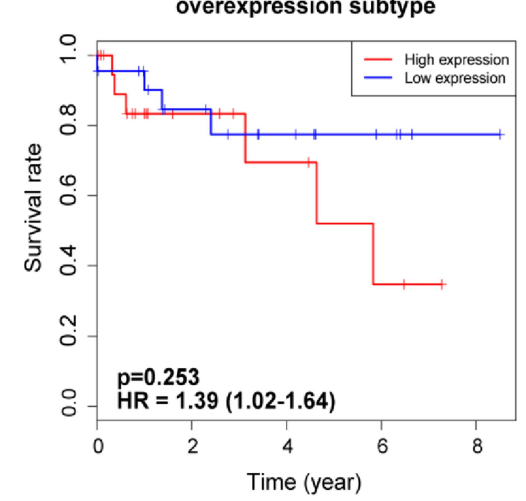

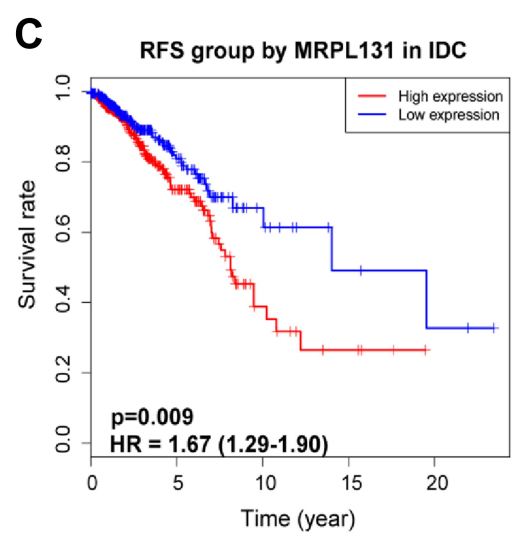

$\mathbf{F}$

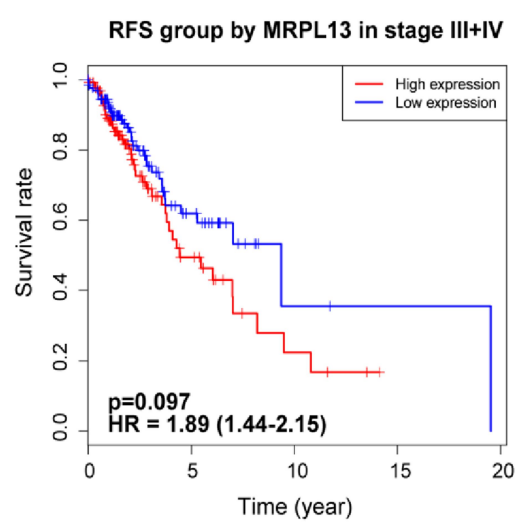

I

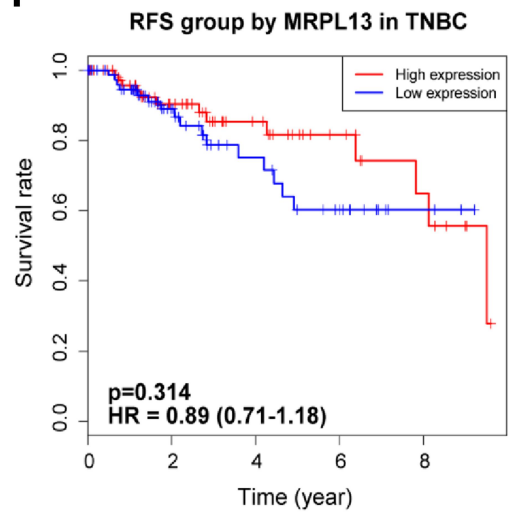

Figure 5 Effect of MRPLI 3 mRNA expression on RFS of BC patients according to different clinical characteristics. Subgroup analysis of group age $<50$ years (A), $\geq 50$ years (B), IDC (C), ILC (D), stage I+II (E), stage III+IV (F), Luminal (G), Her-2 overexpression (H) and TNBC (I) subtypes.

METABRIC database. Twenty-eight percent of those patients harbored a MRLP13 variation, mainly comprising copy number amplification and part of the high and low mRNA status (Figure 6A). As expected, MRPL13 amplification results in the high expression level of MRPL13 (Figure 6B). Compared with the diploid group, the MRPL13 expression level of the gain or amplification group was higher $(p<0.001)$.
Subsequently, we interrogated the differential distribution patterns of MRPL13 CNV in patients with different clinicopathological characteristics, and the results demonstrated that the CNV (gain or amplification) level of MRPL13 gradually increased with clinical stage (S0-25\%, S1-40\%, S2-47\%, S3-56\%, S4-70\%) (Figure 6C), tumor cell proliferation ability (Low-40\%, High-48\%) (Figure 6D) and the tumor grade (G1-22\%, 
Table 5 Univariate and Multivariate Cox Analyses of the Associations Between Prognostic Signatures and Overall Survival in Breast Cancer

\begin{tabular}{|c|c|c|c|c|c|c|}
\hline \multirow[t]{2}{*}{ Clinical Variables } & \multicolumn{3}{|c|}{ Univariate Analysis } & \multicolumn{3}{|c|}{ Multivariate Analysis } \\
\hline & HR & $95 \% \mathrm{Cl}$ & P value & HR & $95 \% \mathrm{Cl}$ & $P$ value \\
\hline Age & 1.61 & $1.12-2.33$ & 0.011 & 1.31 & $0.88-1.94$ & $0.07 I$ \\
\hline Histological type & 1.03 & $0.82-1.98$ & 0.712 & & & \\
\hline Stage & 2.19 & I.74-2.76 & $<0.001$ & 1.90 & $1.62-2.63$ & 0.032 \\
\hline $\mathrm{T}$ & 1.43 & I.I7-1.75 & $<0.001$ & 1.39 & $1.09-1.70$ & 0.097 \\
\hline$N$ & 1.63 & $1.37-1.95$ & $<0.001$ & 1.51 & $1.23-1.88$ & 0.002 \\
\hline M & 4.81 & $2.86-8.07$ & $<0.001$ & 3.98 & $2.01-7.78$ & $<0.001$ \\
\hline ER status & 0.93 & $0.67-1.33$ & 0.129 & & & \\
\hline PR status & 1.02 & $0.76-1.35$ & $0.34 I$ & & & \\
\hline Her-2 status & 1.29 & $0.99-1.87$ & 0.432 & & & \\
\hline TNBC & 0.88 & $0.48-1.5 \mathrm{I}$ & 0.118 & & & \\
\hline MRPLI 3 & 1.98 & $1.19-2.97$ & 0.002 & 1.82 & $1.09-2.38$ & 0.006 \\
\hline
\end{tabular}

Abbreviations: ER, estrogen receptor; PR, progesterone receptor; Her-2, human epidermal growth factor receptor-2; TNBC, triple-negative breast cancer; IDC, infiltrating ductal carcinoma; ILC, infiltrating lobular carcinoma.

G2-37\%, G3-60\%) (Figure 6E). Additionally, the K-M survival analysis clearly indicated that the occurrence of MRPL13 CNV confers significantly shorter OS in $\mathrm{BC}$ patients $(p=1.531 \mathrm{e}-5$, Figure $6 \mathrm{~F})$. The results above imply that patients with CNV (mainly copy number amplification) of MRPL13 are more likely to progress to a late-stage of tumor and suffer from worse prognosis.

\section{Signal Transduction Pathways Associated with MRPLI 3}

To further explore MRPL13-associated signaling pathways in BC, GSEA was conducted to map into KEGG pathway and Cancer Hallmarks databases. The results revealed that significant alterations in tumorous biological behavior were found between high-MRPL13 and low-MRPL13 expression group, as a total of 11 gene sets in Hallmarks and 28 in KEGG were enriched in high-MRPL13 group (Table S1 and S2). Figure 7 illustrates that the top 5 pathways were positively correlated with the expression of MRPL13, including DNA repair, E2F targets, G2M checkpoint, MTORC1 signaling and MYC targets V1 in hallmark (Figure 7A); and cell cycle, Huntington's disease, Oocyte meiosis, RNA degradation and spliceosome in KEGG were also involved (Figure 7B). The GSEA results imply that the high expression of MRPL13 is associated with cell division (meiosis and cell cycle), RNA processing, DNA repair, cancer-related pathways, etc., which may represent an intrinsic mechanism of poor prognosis in $\mathrm{BC}$.

\section{Knockdown of MRPLI 3 Suppresses Proliferation and Migration of BC Cell Lines in vitro}

To confirm whether MRPL13 expression could affect the proliferation and migration of BC cells, MDA-MB-231 and T47D cells were transfected with either control siRNAs (si-NC) or siRNA against MRPL13 (siMRPL13$\mathrm{A} / \mathrm{B} / \mathrm{C}$ ). The qRT-PCR and Western blotting analysis indicated the highest interference efficiency in siMRPL13-A groups for both cell lines, which was consequently used in subsequent experiments (Figure 8A and B). We then examined the effects of MRPL13 silencing on cell proliferation using CCK8 assay. As a result, compared with the negative control, siMRPL13-A treated cells exhibited a prominently lower proliferative capacity in both T47D (Figure $8 \mathrm{C}$, left, $p<0.001$ ) and MDA-MB-231 cell lines (Figure $8 \mathrm{C}$, right, $p<0.001$ ). In addition, wound-healing assay was carried out to evaluate the potential effects of MRPL13 on tumor cell migration (Figure $8 \mathrm{D}$, all $p<0.01$ ). The results showed that silencing of MRPL13 contributed to a marked decrease in migratory ability and motility of MDA-MB-231 and T47D cells. Taken together, these results indicate that MRPL13 is required for BC cell growth and migration.

\section{MRPII 3 Induces EMT Through the AKT/ mTOR Pathway}

As epithelial-mesenchymal transition (EMT) is recognized as a pivotal event coupled with tumor progression, we 


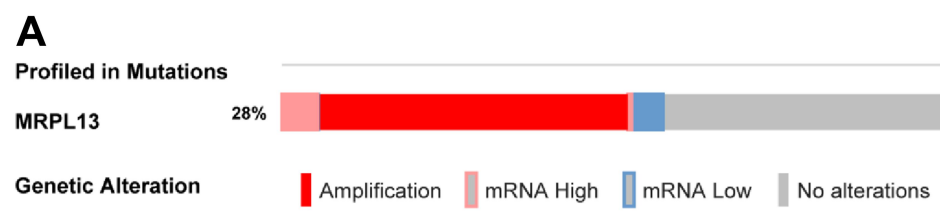

B
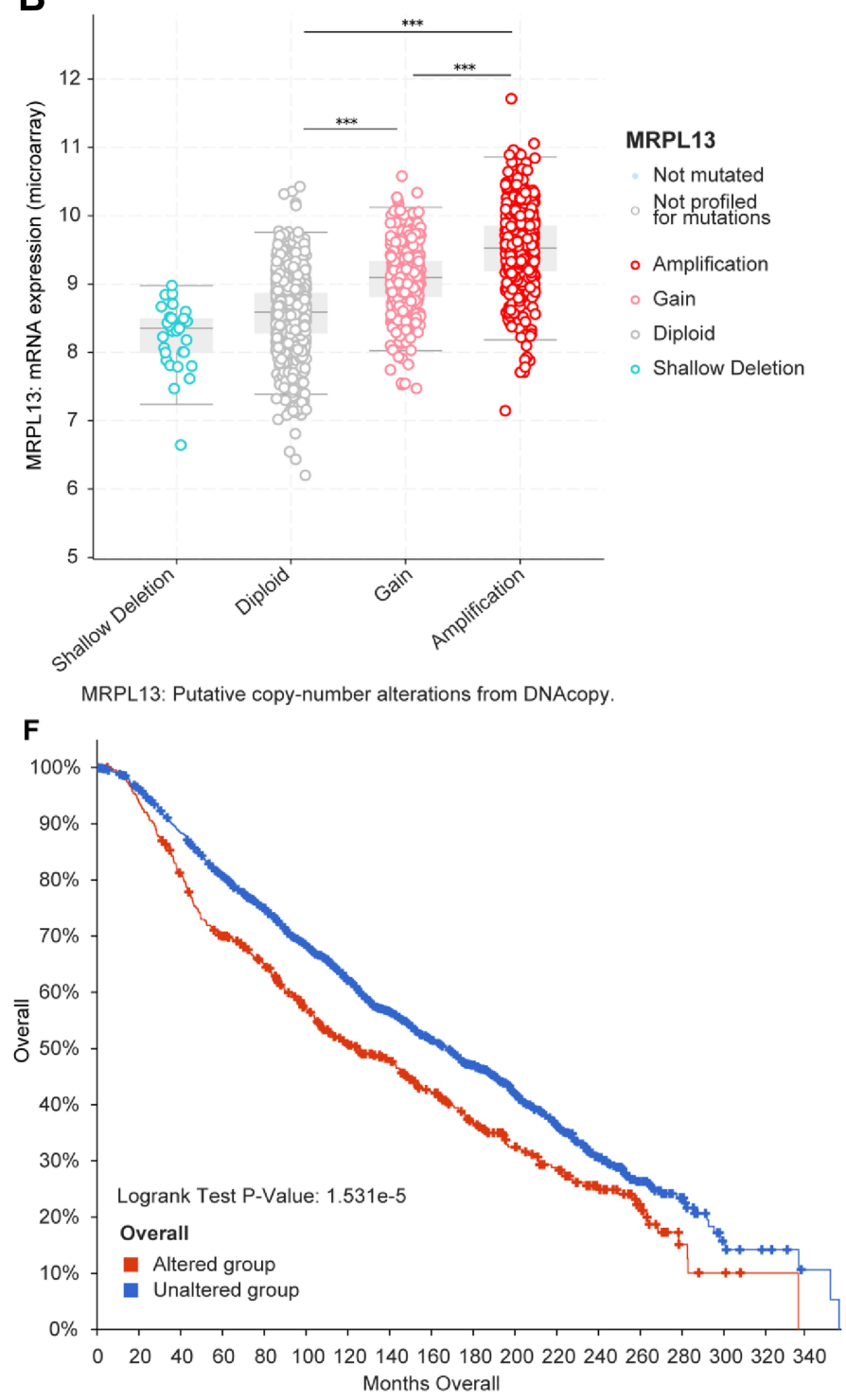
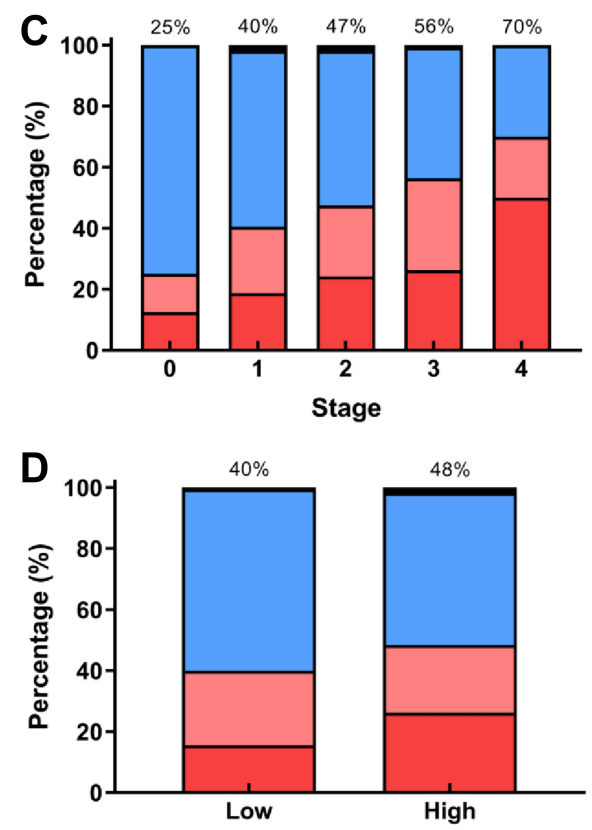

Cell proliferation

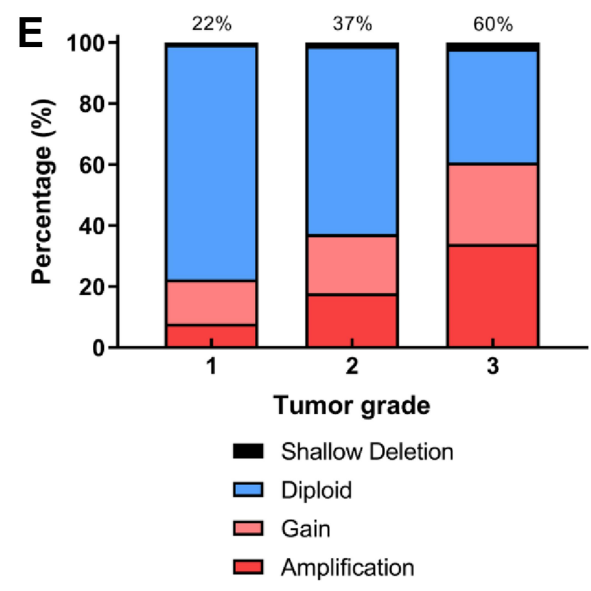

Figure 6 MRPLI3 genomic alterations in BC. (A) Oncoprint of MRPLI3 alterations in METABRIC cohort. The different types of genetic alterations are marked with different colors. (B) Comparison of MRPLI3 expression in different CNV groups. Distribution of MRPLI3 CNV frequency in different grade (C), stage (D) and cell proliferation level (E) subgroups. The percentage number above the bar denotes the proportion of patients with MRPLI3 gain and amplification in this subgroup. (F) Correlation between MRPLI 3 CNV and OS. *** $p<0.001$.

Abbreviations: METABRIC, the Molecular Taxonomy of Breast Cancer International Consortium; CNV, copy number variation.

focused on whether abrogating of MRPL13 in BC cell lines would affect the expression of the EMT-associated molecules. Indeed, the expression of epithelial marker E-cadherin was intensely repressed, whereas mesenchymal marker Vimentin was evidently increased in siMRPL13-A groups for both MDA-MB-231 and T47D cell lines (Figure
9A). Consistently, the expression of EMT-associated transcription factor Snail-1 and Snail-2 was upregulated upon inhibition of MRPL13, further facilitating the EMT inducing potential of MRPL13 in BC (Figure 9A). Subsequently, we moved onto deciphering the mechanism underlying MRPL13-induced EMT process. Based on the above 

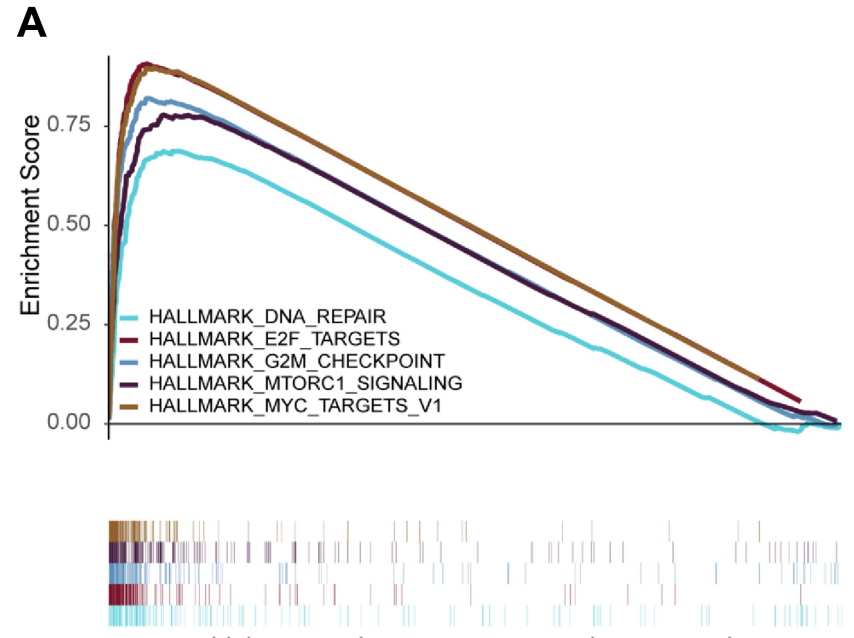

high expression<------------>low expression
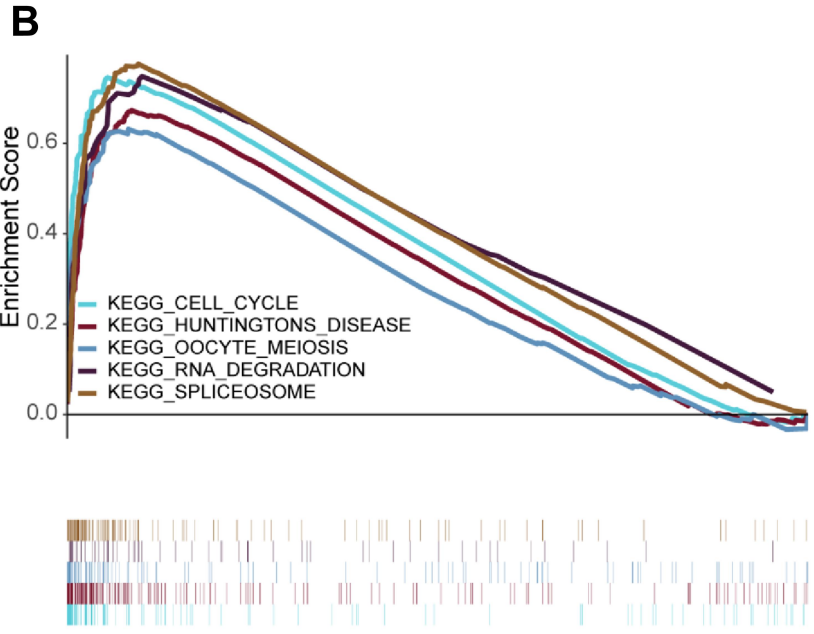

high expression<------------>low expression

Figure 7 Enrichment analysis by GSEA. The top five gene sets enriched in high-MRPLI3 expression group in hallmark (A) and KEGG (B). The different gene sets are highlighted in different colors.

Abbreviations: GSEA, gene set enrichment analysis; KEGG, Kyoto Encyclopedia of Genes and Genomes.

GSEA results, elevated MRPL13 expression was tightly correlated with genes up-regulated in the mTOR signaling canonical pathway (Figure 7A), suggesting that MRPL13 may be involved in the activation of this signaling pathway. As expected, the phosphorylated mTOR (p-mTOR) and phosphorylated AKT (p-AKT), reflecting the bioactivity of mTOR pathway, were markedly decreased in cells with siMRPL13-A treatments, without affecting the total expression levels of these two proteins. Moreover, inhibition of mTOR signaling activity induced by MRPL13 suppression were largely ameliorated with PI3K-specific agonist (740YP) in both MDA-MB-231 and T47D cell lines (Figure 9B and C). These results collectively confirm that MRPL13 promotes proliferation and migration as well as EMT process by triggering activation of the PI3K/AKT/mTOR signaling pathway in $\mathrm{BC}$ cells.

\section{Discussion}

MRPs are functionally responsible for the translation of the oxidative phosphorylation (OXPHOS) complex-related proteins, which provide energy for cell survival and metabolism. ${ }^{27}$ Previous studies have revealed the participation of MRPs in regulating various factors of the intrinsic pathway of apoptosis in carcinomas, such as MRPS29 in hepatocellular carcinoma, MRPL41 in colon cancers, and MRPL65 in ER-positive breast tumors. ${ }^{13,28-30}$ However, it remains ill-defined in the expression states and regulatory mechanism of MRPL13 in cancers, especially in $\mathrm{BC}$.
In the present study, we conducted a series of bioinformatic and statistical approaches to assess the MRPL13 expression profile, its relationship with clinicopathological characteristics, and its impact on clinical outcomes and potential functions in $\mathrm{BC}$, which contributes to a better understanding on the mechanistic details of whether elevated-expressed of specific biomarkers possess any relationship with the discernible clinical survival patterns. We found that MRPL13 expression was pronouncedly increased in BC tissues compared with non-cancerous tissues, inferring a vital regulatory role of this protein in the progression of $\mathrm{BC}$. CNV analysis showed that $\mathrm{BC}$ patients harboring MRPL13 variation mainly arose copy number amplification that led to dysregulated expression, indicating that a high proportion of CNV may be the main factor that causes the increase in MRPL13 expression. Patients with breast tumors in higher grades or in later clinical stages displayed a higher proportion of occurrence of the MRPL13 gene CNV (gain or amplification). These results suggest that the CNV of MRPL13 occurs more frequently with tumor progression and deserves further clinical validation. Additionally, MRPL13 was positively associated with not only advanced T-N-M stages, but also aggressive phenotypes (ER or PR negative and TNBC status) in $\mathrm{BC}$, portending high risks of becoming a more threatening condition of patients with aberrantly elevated MRPL13 expression. More importantly, K-M curves combined with univariate/multivariate model reflected that high expression of MRPL13 could serve as a clinically 
A

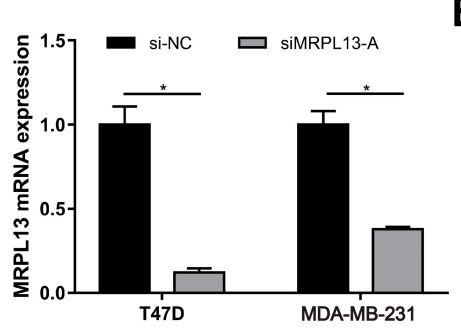

C
B

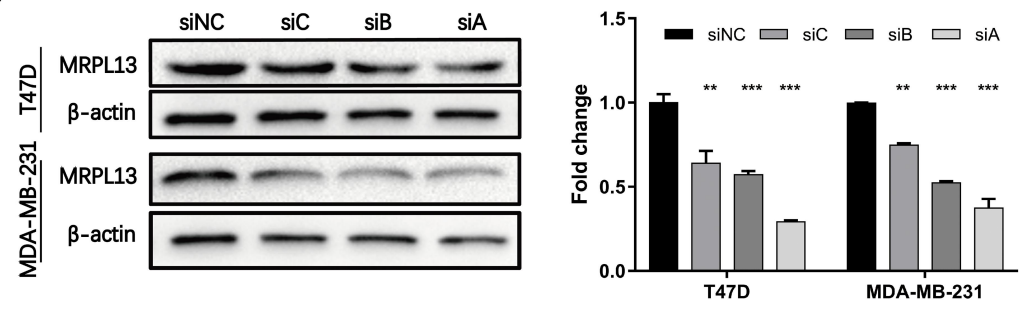

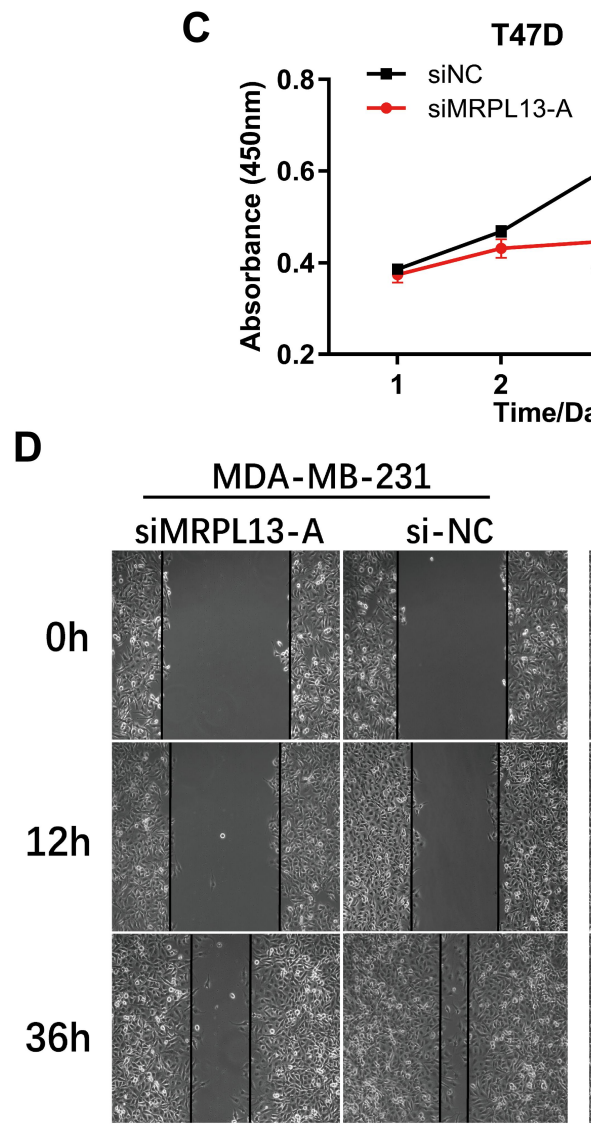

47D

MDA-MB-231

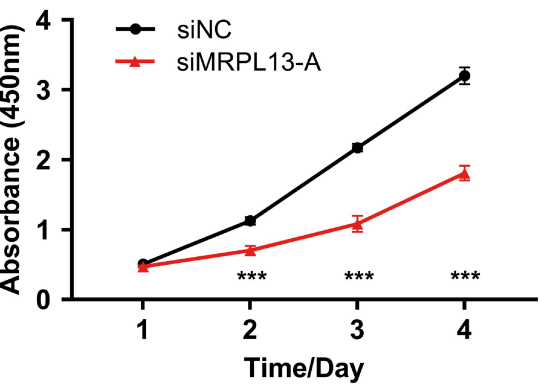

T47D
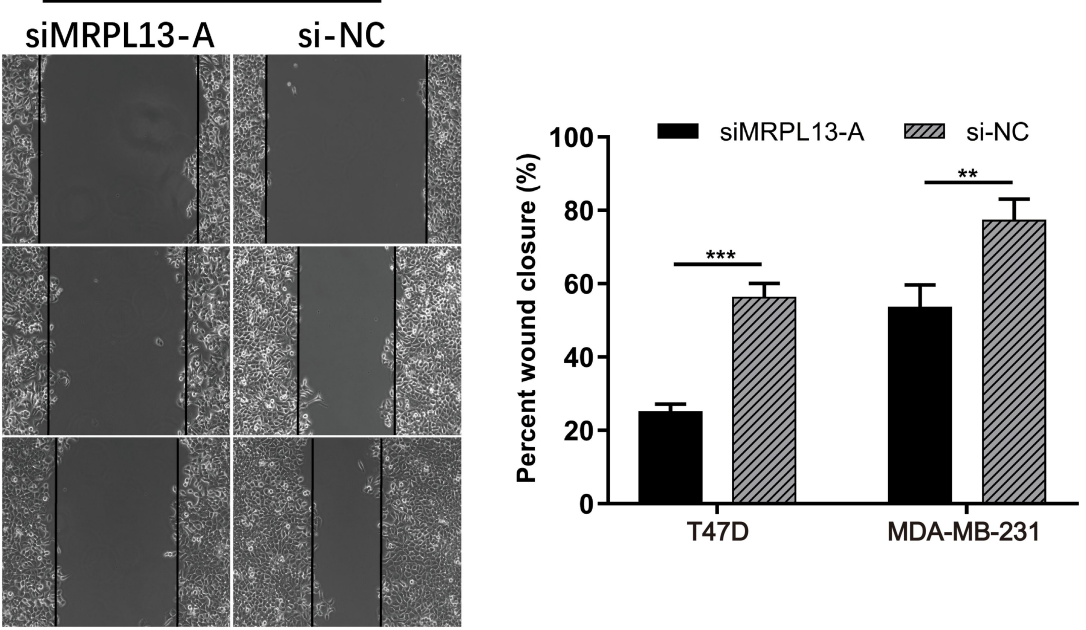

Figure 8 Effects of MRPLI 3 on proliferation and migration of BC cell lines. The Efficiency of siRNA against MRPLI 3 was determined by both qRT-PCR (A) and WB (B) analyses in MDA-MB-23I and T47D cell lines. (C) Cell proliferation was detected by using CCK8 assay in MDA-MB-23I and T47D cells. (D) Cell migration ability was assessed by wound-healing assay in MDA-MB-23I and T47D cells. The $\beta$-actin was used as the internal reference for both qRT-PCR and WB assays. Each test was conducted in triplicate. ${ }^{*} p<0.05 ; * * p<0.01 ; * * * p<0.001$.

Abbreviations: NC, negative control; siRNA, small interfering RNA; qRT-PCR, quantitative real-time PCR; WB, Western blotting; CCK8, cell counting kit-8.

independent prognostic factor in $\mathrm{BC}$. Considering our findings, we cautiously drew the hypothesis that MRPL13 exerts pro-oncogenic effects in BC.

GSEA in this study indicated a close relationship of MRPL13 expression with cell cycle/division-related pathways, RNA processing (degradation/splicing), MYC targets and MTORC1 pathways that were discovered to be involved in the progression of BC. Interestingly, the cyclin gene amplifications were reported to be strongly correlated with BC subtype: CCND1 amplification occurs mainly in ER-positive and HER2-amplified subtypes, while CCNE1 amplification is frequent in TNBC. ${ }^{31}$ Besides, in response to DNA damage, cells activate checkpoints or DNA repair machinery to prevent/eliminate damages. ${ }^{32}$ Our results indicate that the functional consequence of dysregulated expression of MRPL13 mainly includes the disorder of G2/M checkpoint and DNA repair pathway, showing its inhibition in the metabolic processes such as mitochondrial protein synthesis and ribosome biogenesis that are consistent with the molecular pathways implicated in BC carcinogenesis. A prominent example also corroborated with our conclusion, describing that RPS3 accumulation 


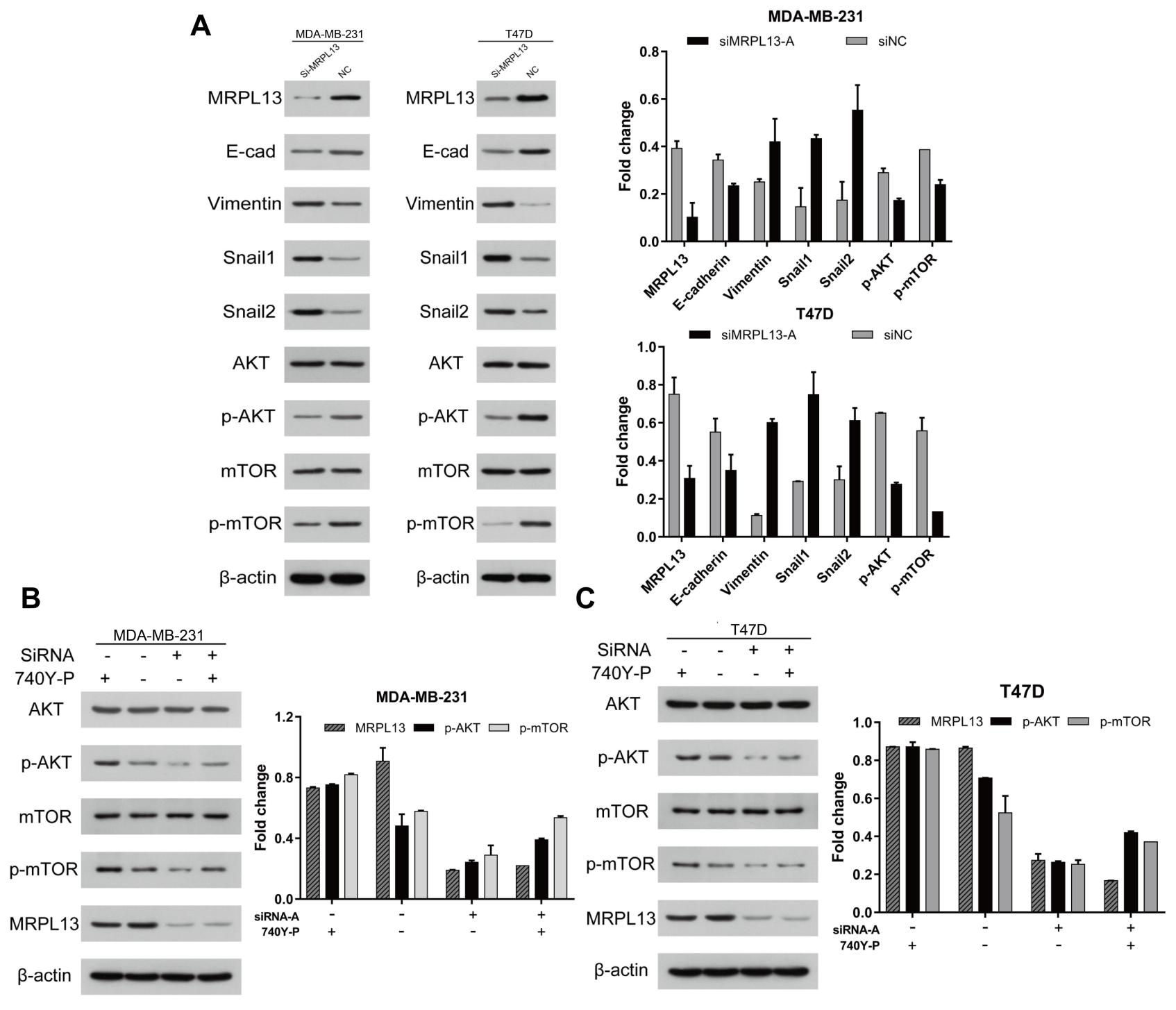

Figure 9 MRPLI3 induces EMT process by activating the Akt/mTOR signaling pathway in MDA-MB-23I and T47D cell lines. MDA-MB-23I and T47D cell lines were transfected with siMRPLI3-A (I00 $\mu \mathrm{M})$ for $72 \mathrm{hr}$. (A) The protein level of EMT-related proteins (E-cad, Vimentin, Snail-I and Snail-2) and AKT/mTOR pathway associated proteins (AKT, p-AKT, mTOR, p-mTOR) was detected by Western blot. For MRPLI 3 siRNA mediated knockdown rescue experiment, cells were transfected independently or jointly with the siMRPLI3-A (I00 $\mu \mathrm{M})$ and PI3K/AKT/mTOR-specific agonist 740Y-P $(20 \mu \mathrm{g} / \mathrm{mL})$. The expression of mTOR signaling and EMT-related proteins was then assessed by Western blot in MDA-MB-23I (B) and T47D (C) cell lines. The $\beta$-actin served as a loading control for all above experiments. The above data are representative of three independent experiments.

Abbreviations: EMT, epithelial-mesenchymal transition; NC, negative control; E-cad, E-cadherin.

caused by the increased ROS level in the mitochondria for DNA repair while significantly impacting the cellular protein synthesis. ${ }^{33}$ In addition, we revealed that MRPL13 may be implicated in the regulation of MYC, one of the most common drivers inducing human cancer through overexpression or hyperactivation. A previous study has suggested the MRPs were up-regulated in MYC-driving lymphoma, indicating a synergetic activation of the mitochondrial translation. Recent research also discovered the spliceosome as a new target of oncogenic stressed in
MYC-driven $\mathrm{BC}$ through a collateral stress given from the increase in total pre-mRNA synthesis induced by MYC hyperactivation. ${ }^{34}$ Drawing on these findings from GSEA that the high expression of MRPL13 is related to a wide range of biological behaviors of tumors, we hypothesized that MRPL13 may directly activate the MYC signaling pathway or potentiate MYC through spliceosomes, and then influence the tumor progression. In addition to these pathways mentioned above, we also tested the effects of MRPL13 on the cancer-related innate 
immune activation, which was previously reported to function in BC progression. ${ }^{35}$

To explore the potential regulation mechanism of MRPL13 in $\mathrm{BC}$, we knockdown the expression of MRPL13 by using small interfering RNA (siRNA) technique for the affirmation of importance of this gene in BC cells. The functional assays indicated that MRPL13 suppression pronouncedly attenuated the proliferation and migration abilities of $\mathrm{BC}$ cells through regulation of the EMT process and AKT/mTOR signaling pathway. EMT is generally considered to be the obligatory step for tumor progression and metastasis, as the neoplastic cells in infancy-stage malignancies are often in an epitheliallike state, and gradually acquire mesenchymal properties with the accumulation of mutations in both oncogenes and tumorsuppressor genes. This process confers the tumor cells' invasive capabilities to disseminate to distant organs and leads to the formation of metastatic lesions. ${ }^{36,37}$ Previous reports have revealed the inhibition of PI3K/AKT/mTOR and EMT signaling suppressed cancer cells' invasion and migration in lung and breast cancer. ${ }^{38-41}$ In view of the diversity and complexity, the key regulatory molecules or links of the EMT pathway involved with MRPL13 in BC will be focused on our next study. Interestingly, growing evidence indicates that EMT programs promote cancer cell stemness properties acquisition and make cancer treatment more difficult. ${ }^{42,43}$ Consequently, great attention should be paid to whether the manipulation of MRPL13 can influence the maintenance or acquisition of the stemness characteristics of tumor cells in future studies. Taken altogether, the results we presented here demonstrated that MRPL13 are involved in BC cell EMT probably through the PI3K/AKT/mTOR pathways. However, we should frankly acknowledge that the current findings still need further confirmation in vivo.

\section{Conclusion}

In summary, our findings suggest that high MRPL13 expression is not only associated with aggressive clinicopathological phenotype and advanced stage, but also correlated to worse prognosticator in $\mathrm{BC}$. Further, as a possible mechanism, increased MRPL13 expression is associated with cell cycle/division-related pathways, RNA processing (degradation/splicing), MYC targets and MTORC1 pathways. Meanwhile, silencing of MRPL13 dramatically suppresses the proliferation and migration abilities and EMT process via modulating the PI3K/AKT/mTOR pathway. Our data merit further investigation into the potential role of MRPL13 as a novel target in $\mathrm{BC}$ treatment.

\section{Data Sharing Statement}

All the data and analysis methods used in this study have been presented in the article and can be obtained through a reasonable request to the corresponding author.

\section{Acknowledgments}

This study was supported by the National Science Foundation of China (Grant No.31800752).

\section{Author Contributions}

All authors made substantial contributions to conception and design, acquisition of data, or analysis and interpretation of data; took part in drafting the article or revising it critically for important intellectual content; agreed to submit to the current journal; gave final approval of the version to be published; and agree to be accountable for all aspects of the work.

\section{Disclosure}

The authors report no conflicts of interest in this work.

\section{References}

1. Wang S, Wang Y, Yu C. Characterization of the relationship between FLI1 and immune infiltrate level in tumour immune microenvironment for breast cancer. J Cell Mol Med. 2020.

2. Waks AG, Winer EP. Breast cancer treatment. JAMA. 2019;321 (3):316. doi:10.1001/jama.2018.20751

3. Kalimutho M, Nones K, Srihari S, Duijf PHG, Waddell N, Khanna KK. Patterns of genomic instability in breast cancer. Trends Pharmacol Sci. 2019;40(3):198-211. doi:10.1016/j.tips.2019.01.005

4. Zheng M, Zhou Y, Yang X, et al. High GINS2 transcript level predicts poor prognosis and correlates with high histological grade and endocrine therapy resistance through mammary cancer stem cells in breast cancer patients. Breast Cancer Res Treat. 2014;148 (2):423-436. doi:10.1007/s10549-014-3172-7

5. Stefanovic S, Deutsch TM, Riethdorf S, et al. The lack of evidence for an association between cancer biomarker conversion patterns and CTC-status in patients with metastatic breast cancer. Int $J$ Mol Sci. 2020;21(6):2161. doi:10.3390/ijms21062161

6. Alvarez RH, Bechara RI, Naughton MJ, Adachi JA, Reuben JM. Emerging perspectives on mTOR inhibitor-associated pneumonitis in breast cancer. Oncologist. 2018;23(6):660-669. doi:10.1634/theoncologist.2017-0343

7. McKenna M, McGarrigle S, Pidgeon GP. The next generation of PI3K-Akt-mTOR pathway inhibitors in breast cancer cohorts. Biochim Biophys Acta Rev Cancer. 2018;1870(2):185-197.

8. Brufsky AM, Dickler MN. Estrogen receptor-positive breast cancer: exploiting signaling pathways implicated in endocrine resistance. Oncologist. 2018;23(5):528-539. doi:10.1634/theoncologist.20170423

9. Piatyszek MA, Denslow ND, O'Brien TW. RNA binding proteins of the large subunit of bovine mitochondrial ribosomes. Nucleic Acids Res. 1988;16(6):2565-2583. doi:10.1093/nar/16.6.2565

10. Lee Y-K, Lim JJ, Jeoun U-W, et al. Lactate-mediated mitoribosomal defects impair mitochondrial oxidative phosphorylation and promote hepatoma cell invasiveness. J Biol Chem. 2017;292(49):20208-20217. doi:10.1074/jbc.M117.809012 
11. Dass S, Mather MW, Ke H. Divergent mitochondrial ribosomes in unicellular parasitic protozoans. Trends Parasitol. 2020;36 (4):318-321. doi:10.1016/j.pt.2020.01.002

12. Porporato PE, Filigheddu N, Pedro JMB-S, Kroemer G, Galluzzi L. Mitochondrial metabolism and cancer. Cell Res. 2018;28(3):265-280.

13. Kim H-J, Maiti P, Barrientos A. Mitochondrial ribosomes in cancer. Semin Cancer Biol. 2017;47:67-81. doi:10.1016/j.semcancer.2017.04.004

14. Yoo YA, Kim MJ, Park JK, et al. Mitochondrial ribosomal protein L41 suppresses cell growth in association with p53 and p27Kip1. Mol Cell Biol. 2005;25(15):6603-6616. doi:10.1128/MCB.25.15.6603-6616.2005

15. Liu L, Luo C, Luo Y, et al. MRPL33 and its splicing regulator hnRNPK are required for mitochondria function and implicated in tumor progression. Oncogene. 2018;37(1):86-94. doi:10.1038/onc.2017.314

16. Best CJM, Gillespie JW, Yi Y, et al. Molecular alterations in primary prostate cancer after androgen ablation therapy. Clin Cancer Res. 2005;11(19 Pt 1):6823-6834. doi:10.1158/1078-0432.CCR-05-0585

17. Pedersen IS, Thomassen M, Tan Q, et al. Differential effect of surgical manipulation on gene expression in normal breast tissue and breast tumor tissue. Mol Med. 2018;24(1):57. doi:10.1186/ s10020-018-0058-x

18. Dhage S, Ernlund A, Ruggles K, et al. A genomic ruler to assess oncogenic transition between breast tumor and stroma. PLoS One. 2018;13(10):e0205602. doi:10.1371/journal.pone.0205602

19. Clarke C, Madden SF, Doolan P, et al. Correlating transcriptional networks to breast cancer survival: a large-scale coexpression analysis. Carcinogenesis. 2013;34(10):2300-2308. doi:10.1093/carcin/bgt208

20. Kretschmer C, Conradi A, Kemmner W, Sterner-Kock A. Latent transforming growth factor binding protein 4 (LTBP4) is downregulated in mouse and human DCIS and mammary carcinomas. Cell Oncol (Dordr). 2011;34(5):419-434. doi:10.1007/s13402-011-0023-y

21. Kretschmer C, Sterner-Kock A, Siedentopf F, Schoenegg W, Schlag PM, Kemmner W. Identification of early molecular markers for breast cancer. Mol Cancer. 2011;10(1):15. doi:10.1186/1476-4598-10-15

22. Nagy Á, Lánczky A, Menyhárt O, Győrffy B. Validation of miRNA prognostic power in hepatocellular carcinoma using expression data of independent datasets. Sci Rep. 2018;8(1):9227. doi:10.1038/ s41598-018-27521-y

23. Cerami E, Gao J, Dogrusoz U, et al. The cBio cancer genomics portal: an open platform for exploring multidimensional cancer genomics data. Cancer Discov. 2012;2(5):401-404. doi:10.1158/21598290.CD-12-0095

24. Subramanian A, Tamayo P, Mootha VK, et al. Gene set enrichment analysis: a knowledge-based approach for interpreting genome-wide expression profiles. Proc Natl Acad Sci USA. 2005;102 (43):15545-15550. doi:10.1073/pnas.0506580102

25. Livak KJ, Schmittgen TD. Analysis of relative gene expression data using real-time quantitative PCR and the 2(-delta delta $\mathrm{C}(\mathrm{T})$ ) method. Methods. 2001;25(4):402-408. doi:10.1006/meth.2001.1262

26. Zarrei M, MacDonald JR, Merico D, Scherer SW. A copy number variation map of the human genome. Nat Rev Genet. 2015;16 (3): $172-183$. doi: $10.1038 / \operatorname{nrg} 3871$

27. Sotgia F, Whitaker-Menezes D, Martinez-Outschoorn UE, et al. Mitochondria "fuel" breast cancer metabolism: fifteen markers of mitochondrial biogenesis label epithelial cancer cells, but are excluded from adjacent stromal cells. Cell Cycle. 2012;11 (23):4390-4401. doi:10.4161/cc.22777

Cancer Management and Research

\section{Publish your work in this journal}

Cancer Management and Research is an international, peer-reviewed open access journal focusing on cancer research and the optimal use of preventative and integrated treatment interventions to achieve improved outcomes, enhanced survival and quality of life for the cancer patient.
28. Sharma MR, Koc EC, Datta PP, Booth TM, Spremulli LL, Agrawal RK. Structure of the mammalian mitochondrial ribosome reveals an expanded functional role for its component proteins. Cell. 2003;115(1):97-108. doi:10.1016/S0092-8674(03)00762-1

29. Cavdar Koc E, Ranasinghe A, Burkhart W, et al. A new face on apoptosis: death-associated protein 3 and PDCD9 are mitochondrial ribosomal proteins. FEBS Lett. 2001;492(1-2):166-170. doi:10.1016/ S0014-5793(01)02250-5

30. Greber BJ, Ban N. Structure and function of the mitochondrial ribosome. Annu Rev Biochem. 2016;85(1):103-132. doi:10.1146/ annurev-biochem-060815-014343

31. Johnson J, Thijssen B, McDermott U, Garnett M, Wessels LFA, Bernards R. Targeting the RB-E2F pathway in breast cancer. Oncogene. 2016;35(37):4829-4835. doi:10.1038/onc.2016.32

32. Majidinia M, Yousefi B. DNA repair and damage pathways in breast cancer development and therapy. DNA Repair (Amst). 2017;54:22-29. doi:10.1016/j.dnarep.2017.03.009

33. Kim Y, Kim HD, Kim J. Cytoplasmic ribosomal protein S3 (rpS3) plays a pivotal role in mitochondrial DNA damage surveillance. Biochim Biophys Acta. 2013;1833(12):2943-2952. doi:10.1016/j. bbamcr.2013.07.015

34. Hsu TYT, Simon LM, Neill NJ, et al. The spliceosome is a therapeutic vulnerability in MYC-driven cancer. Nature. 2015;525 (7569):384-388. doi:10.1038/nature14985

35. Zhou X, Jiang Z. STING-mediated DNA sensing in cancer immunotherapy. Sci China Life Sci. 2017;60(6):563-574. doi:10.1007/s11427-016-9066-0

36. Nieto MA. Epithelial-mesenchymal transitions in development and disease: old views and new perspectives. Int J Dev Biol. 2009;53 (8-10):1541-1547. doi:10.1387/ijdb.072410mn

37. Nieto MA, Huang RY, Jackson RA, Thiery JP. EMT: 2016. Cell. 2016;166(1):21-45.

38. Li Y, Wang T, Sun Y, et al. p53-Mediated PI3K/AKT/mTOR pathway played a role in ptox-induced EMT inhibition in liver cancer cell lines. Oxid Med Cell Longev. 2019;2019:2531493.

39. Katsuno Y, Meyer DS, Zhang Z, et al. Chronic TGF- $\beta$ exposure drives stabilized EMT, tumor stemness, and cancer drug resistance with vulnerability to bitopic mTOR inhibition. Sci Signal. 2019;12 (570):570. doi:10.1126/scisignal.aau8544

40. Baek SH, Ko J-H, Lee JH, et al. Ginkgolic acid inhibits invasion and migration and TGF- $\beta$-induced EMT of lung cancer cells through pi3k/Akt/mTOR inactivation. $J$ Cell Physiol. 2017;232(2):346-354. doi: $10.1002 /$ jcp. 25426

41. Yang F, Takagaki Y, Yoshitomi Y, et al. Inhibition of dipeptidyl peptidase-4 accelerates epithelial-mesenchymal transition and breast cancer metastasis via the CXCL12/CXCR4/mTOR axis. Cancer Res. 2019;79(4):735-746. doi:10.1158/0008-5472.CAN-18-0620

42. Singh A, Settleman J. EMT, cancer stem cells and drug resistance: an emerging axis of evil in the war on cancer. Oncogene. 2010;29 (34):4741-4751. doi:10.1038/onc.2010.215

43. Mani SA, Guo W, Liao MJ, et al. The epithelial-mesenchymal transition generates cells with properties of stem cells. Cell. 2008;133 (4):704-715. doi:10.1016/j.cell.2008.03.027
The manuscript management system is completely online and includes a very quick and fair peer-review system, which is all easy to use. Visit http://www.dovepress.com/testimonials.php to read real quotes from published authors.

\section{Dovepress}

